\title{
Young measure approach to the weak convergence theory in the calculus of variations and strong materials
}

\author{
Mikhail A. SycheV and NinA Nikolaevna Sycheva
}

\begin{abstract}
Let $L(x, u, v): \Omega \times \mathbb{R}^{m} \times \mathbb{R}^{m \times n} \rightarrow \mathbb{R}$ be a Carathéodory integrand with superlinear growth in $v \in \mathbb{R}^{m \times n}$.

Under these assumptions we clarify conditions on $L$-gradient Young measures which imply validity of the weak convergence theory for the associated integral functional $J(u):=\int_{\Omega} L(x, u(x), D u(x)) d x$. Weak convergence theory includes lower semicontinuity with respect to the weak convergence of Sobolev functions, the convergence in energy property (weak convergence of Sobolev functions and convergence in energy imply the strong convergence of the functions), the integral representation for the relaxed energy and related questions. The results of the weak convergence theory follow from a characterization of gradient Young measures associated with the functional. Then we apply the general theory to establish validity of the weak convergence theory for two new classes of integral functionals. Note that the approach of gradient Young measures to the weak convergence theory has certain advantages comparing with $\Gamma$-convergence approach, since homogeneous $L$-gradient Young measures can be characterized for completely arbitrary extended-valued integrands $L$ with infinite growth.

We also disscus the weak convergence theory for so-called strong materials introduced by the first author. We claim that this theory has a better form for such energies. In particular strong materials with $p(x)$-growth admit this theory without any further assumptions on the function $p(x): \Omega \rightarrow \mathbb{R}$ when previously all experts studied the case when $p(\cdot)$ satisfies Zhikov's condition.

Finally we also suggest five conjectures which aim to attract the attention of specialists to further possible developments in the weak convergence theory and to the role of strong materials in Mathematical Theory of Elasticity.
\end{abstract}

Mathematics Subject Classification (2010): 46E27 (primary); 46E35, 74C50, 26B25, 35J20 (secondary).

This work was partially supported by the grant N 12-01-00390 of the Russian Foundation for Basic Research, by the Integration Project N 30 of SIBERIAN DIVISION OF RAS and by the grant N 15 of Presidium of RAS. The research of the first author in the area of the weak convergence theory via the theory of gradient Young measures was started during his stay in ICTP and SISSA (Trieste, Italia) in 1995-1997 years.

Received October 9, 2013; accepted in revised form June 22, 2014.

Published online February 2016. 


\section{Introduction}

In this paper we discuss the weak convergence theory in the Calculus of Variations.

Let $\Omega \subset \mathbb{R}^{n}$ be an open bounded set with Lipschitz boundary. Let also $L: \Omega \times$ $\mathbb{R}^{m} \times \mathbb{R}^{m \times n} \rightarrow \overline{\mathbb{R}}=\mathbb{R} \cup\{\infty\}$ be a Carathéodory integrand with superlinear growth in $v \in \mathbb{R}^{m \times n}$, i.e. $L(x, u, v) \geq \theta(v)$, where $\theta(v) /|v| \rightarrow \infty$ as $|v| \rightarrow \infty$. Recall that $L$ is called a Carathéodory integrand if for each $\epsilon>0$ there exists a compact subset $\Omega_{\epsilon}$ of $\Omega$ such that meas $\left(\Omega \backslash \Omega_{\epsilon}\right) \leq \epsilon$ and $L: \Omega_{\epsilon} \times \mathbb{R}^{m} \times \mathbb{R}^{m \times n} \rightarrow \overline{\mathbb{R}}$ is a continuous function. The Carathéodory assumption implies that for each $u \in W^{1,1}\left(\Omega ; \mathbb{R}^{m}\right)$ the function $L(x, u(x), D u(x))$ is measurable. Since $L$ is bounded from below we define

$$
J(u)=\int_{\Omega} L(x, u(x), D u(x)) d x
$$

in case $L(x, u(x), D u(x)) \in L^{1}$, and $J(u)=\infty$ otherwise.

The weak convergence theory studies the behaviour of integral functionals (1.1) on Sobolev functions, more precisely on sequences of Sobolev functions that converge weakly in $W^{1,1}$. The basic questions of the theory are: lower semicontinuity with respect to the weak convergence $\left(\liminf _{k \rightarrow \infty} J\left(u_{k}\right) \geq J(u)\right.$ for $u_{k} \rightarrow u$ in $W^{1,1}$, here and everywhere further $\rightarrow$ means the weak convergence), convergence in energy property (the convergences $u_{k} \rightarrow u$ in $W^{1,1}, J\left(u_{k}\right) \rightarrow J(u)<\infty$ for $k \rightarrow \infty$ imply the convergence $u_{k} \rightarrow u$ in $\left.W^{1,1}\right)$, integral representation for the lower semicontinuous envelope $\tilde{J}$ of the functional $J$, where

$$
\tilde{J}:=\inf \left\{\liminf _{k \rightarrow \infty} J\left(u_{k}\right): u_{k} \rightarrow u \text { in } W^{1,1}\right\},
$$

and related questions.

As it is well-known, lower semicontinuity is used to establish first of all the existence result in the minimization boundary-value problem, as suggested by Tonelli already in 1915 [50], the relaxation theorem (the theorem on the integral representation for $\tilde{J}$ ) is used to clarify the attainment/nonattainment issues in case lower semicontinuity fails, as it was first suggested by Bogolubov in 1930 [7], the convergence in energy property is involved in studies of proper convergence in numerical schemes $[7,16]$, convergence issues in regularity theory [17], stability results in the theory of quasiconformal mappings [19] etc.

Historically there were different methods to establish results of the weak convergence theory. The most systematic one is to use $\Gamma$-convergence theory introduced by De Giorgi [15]. The latter theory allows to establish an integral representation for a functional $J$ which is a $\Gamma$-limit of a sequence of integral functional $J_{k}$ defined in $W^{1, p}$. However the integral representation is known only in particular cases when the integrands $L_{k}, k \in \mathbb{N}$, satisfy the condition of $p$-growth

$$
c_{1}|v|^{p}+c_{2} \leq L(x, u, v) \leq c_{3}|v|^{p}+c_{4}, c_{3} \geq c_{1}>0, \quad p>1
$$

or, more generally, of $p-q$ growth for appropriate $p, q>1$, see, e.g., [8,20], or when they satisfy a condition of $p(x)$-growth ( $p$ is replaced by $p(x)$ in (1.2)), 
see $[13,32]$. However physical problems require to study the weak convergence theory for more general classes of integrands. In particular problems of mathematical theory of Elasticity require $L$ to be extended-valued with $L(x, u, v)=\infty$ for det $v \leq 0$ (here $m=n$ ) and with $L\left(x, u, v_{k}\right) \rightarrow \infty$ as det $v_{k} \rightarrow+0$, see [3].

In this paper we want to discuss another approach to the weak convergence theory via application of the theory of gradient Young measures:

Recall the definition of Young measures.

Definition 1.1. Let $\left(v_{x}\right)_{x \in \Omega}$ be a family of probability measures $v_{x}, x \in \Omega$, with the supports in $\mathbb{R}^{l}$. Then $\left(v_{x}\right)_{x \in \Omega}$ is called a Young measure provided there exists a sequence $\xi_{k}: \Omega \rightarrow \mathbb{R}^{l}$ of measurable functions such that for each $\Phi \in C_{0}\left(\mathbb{R}^{l}\right)$ we have

$$
\Phi\left(\xi_{k}\right) \rightarrow^{*}\left\langle\Phi ; v_{(\cdot)}\right\rangle \text { in } L^{\infty}, k \rightarrow \infty .
$$

Here $\langle\Phi ; v\rangle$ is the action of a measure $v$ on a continuous function $\Phi$, i.e. $\langle\Phi ; v\rangle=$ $\int_{\mathbb{R}^{l}} \Phi(v) d v . C_{0}\left(\mathbb{R}^{l}\right)$ is the space of continuous functions converging to zero at infinity.

The advantage of using Young measures is that each sequence bounded in $L^{r}$, with $r>0$, contains a subsequence generating a Young measure and lower semicontinuity holds for each extended-valued Carathéodory integrand which is bounded from below. We state the precise results in Theorems 1.2, 1.3.

Theorem 1.2. Let $\xi_{k}: \Omega \rightarrow \mathbb{R}^{l}$ be a sequence of measurable functions such that

$$
\int_{\Omega} L\left(\xi_{k}(x)\right) d x \leq c<\infty, k \in \mathbb{N},
$$

where $L: \mathbb{R}^{l} \rightarrow \overline{\mathbb{R}}$ is a continuous function with the property $L(v) \rightarrow \infty$ as $|v| \rightarrow \infty$.

Then there exists a subsequence $\xi_{k}$ (not relabelled) which generate a Young measure $\left(v_{x}\right)_{x \in \Omega}$.

For a proof see, e.g., $[4,35]$.

Theorem 1.3. Let $\xi_{k}: \Omega \rightarrow \mathbb{R}^{l}$ be a sequence of measurable functions which generate a Young measure $\left(v_{x}\right)_{x \in \Omega}$. Let also $L: \Omega \times \mathbb{R}^{l} \rightarrow \overline{\mathbb{R}}$ be a Carathéodory integrand bounded from below.

Then

$$
\liminf _{k \rightarrow \infty} J\left(\xi_{k}\right) \geq \int_{\Omega}\left\langle L(x, \cdot) ; v_{x}\right\rangle d x,
$$

where in case the right-hand side is finite, the convergence

$$
J\left(\xi_{k}\right) \rightarrow \int_{\Omega}\left\langle L(x, \cdot) ; v_{x}\right\rangle d x<\infty
$$

holds if and only if the sequence $L\left(\cdot, \xi_{k}(\cdot)\right)$, for $k \in \mathbb{N}$, is equiintegrable. In this case we also have

$$
L\left(\cdot, \xi_{k}(\cdot)\right) \rightarrow\left\langle L(\cdot, v) ; \nu_{(\cdot)}\right\rangle \text { in } L^{1}, k \rightarrow \infty .
$$

For a proof of this theorem see, e.g., $[5,36]$. 
To apply Young measure approach to functionals (1.1) we need to study those Young measures which are generated by the gradients, i.e. we need the following:

Definition 1.4. Let $\left(v_{x}\right)_{x \in \Omega}$ be a Young measure with supports in $\mathbb{R}^{m \times n}$ and let $L: \Omega \times \mathbb{R}^{m} \times \mathbb{R}^{m \times n} \rightarrow \mathbb{R}$ be a Carathéodory integrand with at least linear growth in $v \in \mathbb{R}^{m \times n}$. Then $\left(v_{x}\right)_{x \in \Omega}$ is called an $L$-gradient Young measure provided there exists a sequence $u_{k} \in W^{1,1}\left(\Omega ; \mathbb{R}^{m}\right)$ which converges weakly in $W^{1,1}$ to $u_{0} \in W^{1,1}$ such that the gradients $D u_{k}$ generate $\left(v_{x}\right)_{x \in \Omega}$ as a Young measure and

$$
J\left(u_{k}\right) \rightarrow \int_{\Omega}\left\langle L\left(x, u_{0}(x), \cdot\right) ; v_{x}\right\rangle d x<\infty .
$$

Note that because of Theorem 1.3 the convergence property implies that $L\left(\cdot, u_{k}(\cdot)\right.$, $\left.D u_{k}(\cdot)\right), k \in \mathbb{N}$, are equiintegrable and that

$$
L\left(\cdot, u_{k}(\cdot), D u_{k}(\cdot)\right) \rightarrow\left\langle L\left(\cdot, u_{0}(\cdot), v\right) ; v_{(\cdot)}\right\rangle \text { in } L^{1}, k \rightarrow \infty .
$$

In case $L$ satisfies the assumptions of $p$-growth (1.2) $L$-gradient Young measures have a more traditional title of $p$-gradient Young measures.

We also need a definition of homogeneous $L$-gradient Young measures.

Definition 1.5. Let $v$ be a probability measure with support in $\mathbb{R}^{m \times n}$ and let $L$ : $\mathbb{R}^{m \times n} \rightarrow \overline{\mathbb{R}}$ be a continuous integrand with at least linear growth. Let $A \in \mathbb{R}^{m \times n}$ be the center of mass of $v$ and assume that $\langle L ; v\rangle<\infty$. Then $v$ is called a homogeneous $L$-gradient Young measure provided there exists a sequence $u_{k} \in$ $l_{A}+W_{0}^{1, \infty}\left(\Omega ; \mathbb{R}^{m}\right)\left(l_{A}\right.$ is a linear function with the gradient equal to $\left.A\right)$ such that $D u_{k}$ generates $v$ as a homogeneous Young measure and

$$
J\left(u_{k}\right) \rightarrow\langle L ; v\rangle \text { meas } \Omega .
$$

Note that this definition does not depend on $\Omega$. Note also that due to Theorem 1.3 the convergence property in the definition of homogeneous $L$-gradient Young measures implies that $D u_{k} \rightarrow A$ in $L^{1}$.

In the case of integrands with $p$-growth (1.2) $p$-gradient Young measures were characterized by Kinderlehrer and Pedregal in [24].

Theorem 1.6. Let $\left(v_{x}\right)_{x \in \Omega}$ be a Young measure. Then $\left(v_{x}\right)_{x \in \Omega}$ is a p-gradient Young measure if and only if the following three conditions are satisfied:

(i) the center of mass of $\left(v_{x}\right)_{x \in \Omega}$ is the gradient of a Sobolev function $u \in$ $W^{1, p}\left(\Omega ; \mathbb{R}^{m}\right)$;

(ii) for a.e. $x \in \Omega$ the measure $v_{x}$ is a homogeneous p-gradient Young measure;

(iii) $\int_{\Omega}\left\langle|\cdot|^{p} ; v_{x}\right\rangle d x<\infty$.

Kinderlehrer and Pedregal also indicated a characterization of homogeneous $p$ gradient Young measures. It turned out that a probability measure $v$ with the center 
of mass at $A \in \mathbb{R}^{m \times n}$ and with $\left\langle|\cdot|^{p} ; v\right\rangle<\infty$ is a homogeneous $p$-gradient Young measure if and only if Jensen's inequality

$$
\langle L ; v\rangle \geq L(A)
$$

holds for all quasiconvex functions $L$ with $p$-growth (1.2). Recall that $L$ is called quasiconvex at $A$ if for each $\phi \in W_{0}^{1, \infty}\left(\Omega ; \mathbb{R}^{m}\right)$ we have

$$
\int_{\Omega} L(A+D \phi(x)) d x \geq L(A) \text { meas } \Omega .
$$

$L$ is called quasiconvex if it is quasiconvex at each $A$, see [29].

A typical way to construct a quasiconvex function is to consider a quasiconvexification of a continuous function bounded from below. For a continuous function $L: \mathbb{R}^{m \times n} \rightarrow \overline{\mathbb{R}}$ which is bounded from below the quasiconvexification $L^{q c}$ is defined as follows

$$
L^{q c}(A):=\frac{1}{\operatorname{meas} \Omega} \inf _{\phi \in W_{0}^{1, \infty}\left(\Omega ; \mathbb{R}^{m}\right)} \int_{\Omega} L(A+D \phi(x)) d x .
$$

It is known that $L^{q c}$ is a continuous quasiconvex function in case $L$ is finite-valued continuous function which is bounded from below, see, e.g., [37]. Recall that first this result was established by Dacorogna [14] for special $L$.

In [38] we precised the characterization result and proved that it is enough to verify the inequality (1.3) with quasiconvex $L$ of the form $|\cdot|^{p}+\Phi(\cdot)$, where $\Phi \in C_{c}\left(\mathbb{R}^{m \times n}\right)$ (here the index "c" means compactness of the support of $\Phi$ ).

Moreover Kinderlehrer and Pedregal established in [24,25] that Young measures generated by the gradients of Sobolev functions bounded in $W^{1, p}$, with $p>1$, are $p$-gradient Young measures.

Theorem 1.7. Let $\left(v_{x}\right)_{x \in \Omega}$ be a Young measure generated by the gradients of a sequence $u_{k}$ which is bounded in $W^{1, p}, p>1$. Then $\left(v_{x}\right)_{x \in \Omega}$ satisfies the conditions (i)-(iii) of Theorem 1.6.

Independently Kristensen proved Theorems 1.6, 1.7 in [26]. He also observed that Theorem 1.7 is a consequence of a more general fact on Sobolev functions. It turned out that given a sequence $u_{k}$ bounded in $W^{1, p}, p>1$, there exists a subsequence $u_{k}$ (not relabelled) and a sequence $w_{k} \in W^{1, p}$ such that $\left|D w_{k}\right|^{p}$ are equiintegrable and $\left|D w_{k}-D u_{k}\right| \rightarrow 0$ in $L^{1}$. As a consequence $D w_{k}$ and $D u_{k}$ generate the same Young measure which is automatically a $p$-gradient Young measure because of the equiintegrability of $\left|D w_{k}\right|^{p}, k \in \mathbb{N}$.

Theorems 1.6, 1.7 make the approach of gradient Young measures attractive for studying the questions of the weak convergence theory (in the case of integrands with $p$-growth). In $[36,39]$ we showed how to derive optimal results of the weak convergence theory for this class of integrands via Theorems 1.6, 1.7. These results are sharper than those previously available. 
To apply the Young measure approach to the weak convergence theory we also needed to develop certain new technical tools for Young measures. In particular it turned out convenient to consider Young measures $\left(v_{x}\right)_{x \in \Omega}$ as mappings to a metric space $\left(M_{1}, \rho\right)$, where $M_{1}$ is the set of all measures bounded in total variation by 1 and $\rho$ is the metric characterizing weak* convergence of measures, i.e.

$$
\rho(\nu, \mu):=\sum_{i=1}^{\infty} \frac{1}{2^{k}\left\|\Phi_{k}\right\|_{C}}\left|\left\langle\Phi_{k} ; v\right\rangle-\left\langle\Phi_{k} ; \mu\right\rangle\right|,
$$

where $\Phi_{k} \in C_{c}^{\infty}\left(\mathbb{R}^{l}\right)$, for $k \in \mathbb{N}$, is a dense sequence in the space $C_{0}\left(\mathbb{R}^{l}\right)$. It turned out that $\left(v_{x}\right)_{x \in \Omega}$ is a Young measure if and only if $v: \Omega \rightarrow\left(M_{1}, \rho\right)$ is a measurable function. Such functions have the Lusin property, i.e. for each $\epsilon>0$ there exists a subset $\Omega_{\epsilon}$ of $\Omega$ such that meas $\left(\Omega \backslash \Omega_{\epsilon}\right) \leq \epsilon$ and $\nu: \Omega_{\epsilon} \rightarrow\left(M_{1}, \rho\right)$ is continuous. The continuity property is convenient to derive various results on general Young measures via a.e. approximation in $\rho$-metric of general Young measures by more regular ones which are easier to construct. Another tool suggested was a method to construct a Young measure $\left(v_{x}\right)_{x \in \Omega}$ from homogeneous Young measures $v_{x} \in$ $V(x) \subset\left(M_{1}, \rho\right), x \in \Omega$. This tool is a selection theorem for measurable multivalued mappings $V: \Omega \rightarrow 2^{\left(M_{1}, \rho\right)}$. In [36] we mentioned that the characterization of Young measures as measurable mappings $v: \Omega \rightarrow\left(M_{1}, \rho\right)$, the form of the metric and the tools just described are in fact a new approach to general Young measure theory since the standard results of Young measure theory can be derived via this approach. We did not publish the related proofs in [36] due to restrictions on the length of papers and these proofs appeared only much later in [35].

Though the papers $[36,39]$ showed how to address the issues of the weak convergence theory via Young measures it remained unclear how to use this approach in more general problems since the characterization result (Theorem 1.6) from [24] relied severely on the assumptions of $p$-growth. In [38] we applied our approach to Young measures as to measurable functions with values in $\left(M_{1}, \rho\right)$ and showed that homogeneous $L$-gradient Young measures can be characterized for any continuous integrand $L: \mathbb{R}^{m \times n} \rightarrow \overline{\mathbb{R}}$ with at least linear growth. It turned out that the following theorem is valid

Theorem 1.8. Let $L: \mathbb{R}^{m \times n} \rightarrow \overline{\mathbb{R}}$ be a continuous integrand with at least linear growth and let $v$ be a probability measure with the support in $\mathbb{R}^{m \times n}$, with the center of mass at $A$ and with finite action on $L$.

Then $v$ is a homogeneous L-gradient Young measure if and only if for each $\Phi \in C_{c}^{\infty}\left(\mathbb{R}^{m \times n}\right)$ the inequality

$$
\langle L+\Phi ; v\rangle \geq(L+\Phi)^{q c}(A)
$$

holds.

In [38] we did not include any results on nonhomogeneous $L$-gradient Young measures and this, might be, was a reason why the approach of gradient Young measures did not attract the attention of other researchers in the field. Instead, during the last decade we studied separately several classes of integrands for which 
the weak convergence theory could be developed via this approach. In each of these cases analogs of Theorems 1.6, 1.7 were established and then the schemes of the papers $[36,39]$ were applied to establish results of the weak convergence theory. This way we studied so-called strong materials

$$
L(x, u, v) \geq \alpha|v|^{n+\epsilon}+\beta, \text { for } \alpha>0, \epsilon>0,
$$

in the scalar case $m=1$. It turned out that the relaxation theorem is available for Carathéodory integrands $L: \Omega \times \mathbb{R} \times \mathbb{R}^{n} \rightarrow \overline{\mathbb{R}}$ of type (1.4) only under the assumption that $L$ is bounded in a neighbourhood of each point where it is finite, [40]. Then we studied the case of integrands with fast growth (faster than the polynomial one) in [41]. We established lower semicontinuity and relaxation results for this case, i.e. when

$$
c_{1} G(|v|)+c_{2} \leq L(x, u, v) \leq c_{3} G(|v|)+c_{4}, c_{3} \geq c_{1}>0
$$

where $G(t):[0, \infty[\rightarrow[0, \infty[$ is such a nondecreasing convex function that $G^{\prime}(t) t / G(t) \rightarrow \infty$ monotonically as $t \rightarrow \infty$. Finally in [42,43] we established the results of the weak convergence theory for the case of integrands with $p(x)$-growth

$$
c_{1}|v|^{p(x)}+c_{2} \leq L(x, u, v) \leq c_{3}|v|^{p(x)}+c_{4}, c_{3} \geq c_{1}>0,
$$

where $1<p_{1} \leq p(\cdot) \leq p_{2}<\infty$ and $p(\cdot)$ satisfies Zhikov's condition. These results are sharper than those available via $\Gamma$-convergence theory, see $[13,32]$.

Note also that the weak convergence theory was complemented by one more general result which states that for an arbitrary Carathéodory integrand $L: \Omega \times$ $\mathbb{R}^{m} \times \mathbb{R}^{m \times n} \rightarrow \overline{\mathbb{R}}$ the set where the functional is both lower semicontinuous and admits the convergence in energy property is dense with respect to the weak topology in the set of definition of the functional, more precisely the following theorem from [44] is valid.

Theorem 1.9. Let $L(x, u, v): \Omega \times \mathbb{R}^{m} \times \mathbb{R}^{m \times n} \rightarrow \overline{\mathbb{R}}$ be a Carathéodory integrand with superlinear growth in $v \in \mathbb{R}^{m \times n}$. Let also $S$ be a subset of $W^{1,1}$ which contains all limit points $u \in W^{1,1}$ of sequences $u_{k} \in S$ which converge strongly in $W^{1,1}$ and have the property $\lim \sup _{k \rightarrow \infty} J\left(u_{k}\right)<\infty$. Assume also that for each $c>0$ the set $S_{c}:=\{u \in S: J(u) \leq c\}$ is weakly precompact in $W^{1,1}$ (in particular $S$ could be the set of $u \in W^{1,1}$ satisfying the boundary condition $\left.\left.u\right|_{\partial \Omega}=f\right)$.

Then the set $V \subset S^{\prime}$ (here $S^{\prime}=\{u \in S: J(u)<\infty\}$ ) where the functional $J: S^{\prime} \rightarrow \mathbb{R}$ is both lower semicontinuous and admits the convergence in energy property is dense in $S^{\prime}$ in the weak topology. The functional $\tilde{J}: \bar{S}^{\prime} \rightarrow \overline{\mathbb{R}}\left(\bar{S}^{\prime}\right.$ is the closure of $S^{\prime}$ in the weak topology of $W^{1,1}$ ) which is the lower semicontinuous extension of the functional $J: S^{\prime} \rightarrow \mathbb{R}$ with respect to the weak topology of $W^{1,1}$ also admits the convergence in energy property at the elements of $V$. Moreover, given $u \in \bar{S}^{\prime}$ with $\tilde{J}(u)<\infty$ there exists a sequence $u_{k} \in V$ such that $u_{k} \rightarrow u$ in $W^{1,1}$ and $J\left(u_{k}\right) \rightarrow \tilde{J}(u), k \rightarrow \infty$. 
This result was obtained as a development of the approach to study maximal oscillations produced by admissible functions weakly convergent to a given one originated in context of theory of differential inclusions in [48].

Now it is logical to address the abstract case of Carathéodory integrands with the superlinear growth in $D u$ and to describe which properties of the associated $L$ gradient Young measures are sufficient to claim validity of the weak convergence theory for the associated functional instead of studying separately new classes of integrands, i.e. we want to establish general method of gradient Young measures to study the weak convergence theory. To do this we need to understand which Young measures are $L$-gradient Young measures in the case of arbitrary integrands.

The following theorem is valid:

Theorem 1.10. Let $L(x, u, v): \Omega \times \mathbb{R}^{m} \times \mathbb{R}^{m \times n} \rightarrow \mathbb{R}$ be a Carathéodory integrand with the superlinear growth in $v \in \mathbb{R}^{m \times n}$ and assume that $L$ is bounded on compact sets. Let $\left(v_{x}\right)_{x \in \Omega}$ be a Young measure. Then $\left(v_{x}\right)_{x \in \Omega}$ is an L-gradient Young measure provided the following four conditions (h1)-(h4) are valid:

(h1) center of mass of $\left(v_{x}\right)_{x \in \Omega}$ is the gradient of a Sobolev function $u_{0} \in W^{1,1}\left(\Omega ; \mathbb{R}^{m}\right)$ with $J\left(u_{0}\right)<\infty$;

(h2) for a.e. $x \in \Omega$ the measure $v_{x}$ is a homogeneous $L\left(x, u_{0}(x), \cdot\right)$-gradient Young measure;

(h3) $\int_{\Omega}\left\langle L\left(x, u_{0}(x), \cdot\right) ; v_{x}\right\rangle d x<\infty$;

(h4) there exists an almost piece-wise affine sequence $u_{k} \in W^{1,1}, k \in \mathbb{N}$, such that $u_{k} \rightarrow u_{0}$ in $W^{1,1}$ and $J\left(u_{k}\right) \rightarrow J\left(u_{0}\right)$ as $k \rightarrow \infty$.

A sequence $w_{k} \in W^{1,1}$ whose gradients generate $\left(v_{x}\right)_{x \in \Omega}$ as a L-gradient Young measure can be taken in the form $w_{k} \in u_{k}+W_{0}^{1, \infty}\left(\Omega ; \mathbb{R}^{m}\right), k \in \mathbb{N}$.

In the scalar case $m=1$ this result remains valid for extended-valued Caratheodory integrands $L: \Omega \times \mathbb{R} \times \mathbb{R}^{n} \rightarrow \overline{\mathbb{R}}$ which are bounded in a neighbourhood of each point where they are finite.

Here we say that $u_{k} \in W^{1,1}\left(\Omega ; \mathbb{R}^{m}\right)$, for $k \in \mathbb{N}$, is an almost piece-wise affine sequence of functions provided there exist open sets $\Omega_{k} \subset \Omega, k \in \mathbb{N}$, such that meas $\left(\Omega \backslash \Omega_{k}\right) \rightarrow 0$ as $k \rightarrow \infty$ and the restrictions of $u_{k}$ to $\Omega_{k}, k \in \mathbb{N}$, are piece-wise affine.

The result of Theorem 1.10 could be established for the case of extendedvalued Carathéodory integrands $L: \Omega \times \mathbb{R}^{m} \times \mathbb{R}^{m \times n} \rightarrow \overline{\mathbb{R}}$ in the general vectorial case $(m>1)$ provided that in Definition 1.5 of homogeneous $L$-gradient Young measures the sequence $u_{k} \in l_{A}+W_{0}^{1, \infty}\left(\Omega ; \mathbb{R}^{m}\right)$ is taken with the additional property $L\left(D u_{k}\right) \leq M(k)<\infty$ a.e. in $\Omega, k \in \mathbb{N}$. In the scalar case $m=1$ this property holds automatically and this is why Theorem 1.10 holds in the scalar case for extended-valued integrands. In this paper we prefer not to treat the case of extended-valued integrands in the vectorial situation since this is a separate subtle issue which we hope to address somewhere later.

Theorem 1.10 could be stated already in [38] where we addressed the problem of characterizing homogeneous $L$-gradient Young measures. However at that time 
it was not appropriate to suggest an abstract approach to the weak convergence theory since no other examples but the case of $p$-growth were available. Now the experience accumulated naturally suggests to develop the abstract approach which is the main subject of this paper. Then new classes of functionals, see Section 6, can be addressed from this general point of view.

Note that the cases available for the analysis are such that Young measures $\left(v_{x}\right)_{x \in \Omega}$ generated by gradients of sequences bounded in energy satisfy conditions (h1)-(h4), i.e. they are $L$-gradient Young measures, see next section. We state the results of this paper in the next section. In Section 3 we describe our approach to Young measures as to measurable functions $v: \Omega \rightarrow\left(M_{1}, \rho\right)$ as it was suggested in [35]. In Section 4 we prove Theorem 1.10 and in Section 5 we prove the main result of Section 2. Then in Section 6 we apply general result of Section 2 to two new cases of functionals which we describe at the end of Section 2. Note that one of these cases is the case of strong materials, see (1.4), with $p(x)$-growth which was intensely studied in the literature from the last decade.

ACKNOWLEDGEMENTS. The first author benefited from interaction with professors of SISSA when developing Young measure approach to the weak convergence theory during his stay in ICTP and SISSA (both in Trieste, Italy) in 1995-1997 years.

We thank Andrea Braides, Alberto Bressan, Arrigo Cellina and Gianni Dal Maso for useful and friendly scientific interaction. The research in the field was continued in Carnegie Mellon University (Pittsburgh, USA) and in the Sobolev Institute of Mathematics (Novosibirsk, Russia). Both the authors thank the Head of Laboratory of Differential Equations and Related Problems in Analysis of the Sobolev Institute Prof. Vladimir Belonosov for his interest in it and the support given. It is also a great pleasure to thank Academician Yurii Reshetnyak for his interest to the research and for his support.

Part of results of this paper was obtained while the first author visited University of Nimes (Nimes, France) in May-June of 2011 year. For this reason he thanks Jean-Philippe Mandallena and Anza Hafsa Omar for benefitial scientific interaction.

We thank Jan Kristensen for many useful consultations.

The authors thank the unknown referee for useful remarks which allowed to improve the presentation of the paper.

\section{Conditions on gradient Young measures implying the validity of the weak convergence theory}

In this section we state the main results of this paper. We describe assumptions on Young measures generated by gradients of Sobolev functions bounded in energy which imply the validity of the theory of weak convergence for the related integral functional. 
First we state what we mean by weak convergence theory (Statements 2.1-2.5). Statement 2.1. Let $L(x, u, v): \Omega \times \mathbb{R}^{m} \times \mathbb{R}^{m \times n} \rightarrow \mathbb{R}$ be a Carathéodory integrand with superlinear growth in $v \in \mathbb{R}^{m \times n}$ which is bounded on compact sets. Let $u \in W^{1,1}\left(\Omega ; \mathbb{R}^{m}\right)$ be such that $J(u)<\infty$. Then the convergence $u_{k} \rightarrow u$ in $W^{1,1}$ implies the inequality

$$
\liminf _{k \rightarrow \infty} J\left(u_{k}\right) \geq J(u)
$$

if and only if the integrand $L(x, u(x), \cdot)$ is quasiconvex at $D u(x)$ for a.e. $x \in \Omega$.

It is clear that, by Statement 2.1, quasiconvexity of $L(x, u, v)$ in $v$ for a.e. $x \in \Omega$ and for all $u \in \mathbb{R}^{m}$ is both a necessary and sufficient condition for the functional $J(u)$ to be lower semicontinuous with respect to the weak convergence in $W^{1,1}$.

Statement 2.2. Let $L(x, u, v): \Omega \times \mathbb{R}^{m} \times \mathbb{R}^{m \times n} \rightarrow \mathbb{R}$ be a Carathéodory integrand with superlinear growth in $v \in \mathbb{R}^{m \times n}$ and assume that $L$ is bounded on compact sets. Then the function

$$
L^{q c}(x, u, v):=\frac{1}{\operatorname{meas} \Omega} \inf _{\phi \in W_{0}^{1, \infty}\left(\Omega ; \mathbb{R}^{m}\right)} \int_{\Omega} L(x, u, v+D \phi(y)) d y
$$

is a Carathéodory integrand with superlinear growth in $v$, is bounded on compact sets and is quasiconvex in $v$ for a.e. $x \in \Omega$ and all $u \in \mathbb{R}^{m}$.

The lower semicontinuous envelope $\tilde{J}$ of $J$ has the property $\tilde{J}=J^{q c}$, where $J^{q c}=\int_{\Omega} L^{q c}(x, u(x), D u(x)) d x$, in the set $S=\left\{u \in W^{1,1}: J(u)<\infty\right\}$. For other $u \in W^{1,1}$ we have $J^{q c}(u) \leq \tilde{J}(u)$.

Remark 2.3. Usually integrands $L$ satisfy certain assumptions which also hold for $L^{q c}$. If

$$
c_{1} G(x, u, v)+c_{2} \leq L(x, u, v) \leq c_{3} G(x, u, v)+c_{4}, c_{3} \geq c_{1}>0,
$$

where $G$ is convex (or more generally quasiconvex) in $v$, then $L^{q c}$ satisfies the same inequalitites and, since so, $J^{q c}$ and $J$ are finite in the same set. As a consequence $\tilde{J}=J^{q c}$ everywhere.

A continuous function $G: \mathbb{R}^{m \times n} \rightarrow \overline{\mathbb{R}}$ with $G(v) \rightarrow \infty$ as $|v| \rightarrow \infty$ is called strictly quasiconvex at $A \in \mathbb{R}^{m \times n}$ if for any sequence $\phi_{k} \in W_{0}^{1, \infty}\left(\Omega ; \mathbb{R}^{m}\right)$ the convergence

$$
\int_{\Omega} G\left(A+D \phi_{k}(x)\right) d x \rightarrow G(A) \text { meas } \Omega, k \rightarrow \infty,
$$

implies the convergence $D \phi_{k} \rightarrow 0$ in measure. Note that strict quasiconvexity at $A$ implies quasiconvexity at $A$ because of the growth assumptions. 
Statement 2.4. Let $L(x, u, v): \Omega \times \mathbb{R}^{m} \times \mathbb{R}^{m \times n} \rightarrow \mathbb{R}$ be a Carathéodory integrand with superlinear growth in $v \in \mathbb{R}^{m \times n}$ and assume that $L$ is bounded on compact sets. Let also $u \in W^{1,1}$ be such that $J(u)<\infty$.

Then the convergences $u_{k} \rightarrow u$ in $W^{1,1}, J\left(u_{k}\right) \rightarrow J(u)$ for $k \rightarrow \infty$ imply the convergence $u_{k} \rightarrow u$ in $W^{1,1}$ if and only if $L(x, u(x), \cdot)$ is strictly quasiconvex at $D u(x)$ for a.e. $x \in \Omega$.

The last result of the weak convergence theory is the following theorem.

Statement 2.5. Let $L(x, u, v): \Omega \times \mathbb{R}^{m} \times \mathbb{R}^{m \times n} \rightarrow \mathbb{R}$ be a Carathéodory integrand with superlinear growth in $v \in \mathbb{R}^{m \times n}$ and which is bounded on compact sets. Let $f \in W^{1,1}$ be such that $J(f)<\infty$. Consider the set

$$
S=\left\{u \in W^{1,1}: J(u)<\infty,\left.u\right|_{\partial \Omega}=\left.f\right|_{\partial \Omega}\right\} .
$$

Let $\bar{S}$ be a closure of $S$ in the weak topology of $W^{1,1}$. Then the set $V \subset S$ such that for each $u \in V$ the functions $L(x, u(x), \cdot), L^{q c}(x, u(x), \cdot)$ are strictly quasiconvex (and then equal) at $D u(x)$ for a.e. $x \in \Omega$ is dense in $S$ in the weak topology of $W^{1,1}$. For each $u \in \bar{S}$ with $\tilde{J}(u)<\infty$ there exists a sequence $u_{k} \in V$ such that $u_{k} \rightarrow u$ in $W^{1,1}$ and $J\left(u_{k}\right) \rightarrow \tilde{J}(u)$ as $k \rightarrow \infty$.

Now we state conditions on Young measures generated by the gradients of Sobolev functions bounded in energy which imply Statements 2.1-2.5.

1) Let $u_{k} \in W^{1,1}\left(\Omega ; \mathbb{R}^{m}\right)$ be such that $J\left(u_{k}\right) \leq c<\infty, k \in \mathbb{N}$, and $D u_{k}$ generate a Young measure $\left(v_{x}\right)_{x \in \Omega}$ (then $u_{k} \rightarrow u_{0}$ in $W^{1,1}$ because of superlinear growth of $L(x, u, v)$ in $v)$. Then $v_{x}$ is a homogeneous $L\left(x, u_{0}(x), \cdot\right)$-gradient Young measure for a.e. $x \in \Omega$.

Note that equiintegrability of $\left|D u_{k}\right|, k \in \mathbb{N}$, always implies that for a.e. $x \in \Omega$ the measure $v_{x}$ is a homogeneous 1-gradient Young measure. However the requirement that $v_{x}$ is a homogeneous $L\left(x, u_{0}(x), \cdot\right)$-gradient Young measure is sufficiently restrictive and is a key one. We impose this requirement since we want to obtain lower semicontinuity via Jensen's inequality

$$
\left\langle L\left(x, u_{0}(x), \cdot\right) ; v_{x}\right\rangle \geq L\left(x, u_{0}(x), D u_{0}(x)\right)
$$

in case $L\left(x, u_{0}(x), \cdot\right)$ is quasiconvex at $D u_{0}(x)$. For this purpose we need $v_{x}$ to be generated by a sequence $A+D \phi_{k}, k \in \mathbb{N}$, with $\phi_{k} \in W_{0}^{1, \infty}\left(\Omega ; \mathbb{R}^{m}\right)$ having the property of convergence in energy, see Definition 1.5.

In general it is not true that for a.e. $x \in \Omega$ the measures $v_{x}$ are homogeneous $L\left(x, u_{0}(x), \cdot\right)$-gradient Young measures, as well it is not true that quasiconvexity is sufficient for the lower semicontinuity result. A well-known counterexample is due to Ball-Murat, see [6]. In case $m=n$ the integrand

$$
L(v)=\mu|v|^{n-\epsilon}+|\operatorname{Det} v|, \mu>0, \epsilon>0,
$$


is obviously quasiconvex. However it admits the so-called Lavrentiev phenomenon in problem with boundary data $\left.u\right|_{\partial \Omega}=\left.\mathrm{Id}\right|_{\partial \Omega}$, here $\Omega=B(0,1)$. Assume $u(x):=$ $x /|x| \in W^{1, n-\epsilon}$ for all $\epsilon>0$. Then for a fixed sufficiently small $\epsilon>0$ we have

$\inf \left\{J(u): u \in \operatorname{Id}+W_{0}^{1, n-\epsilon}\left(\Omega ; \mathbb{R}^{m}\right)\right\}<L(\operatorname{Id})=\inf \left\{J(u): u \in \operatorname{Id}+W_{0}^{1, \infty}\left(\Omega ; \mathbb{R}^{m}\right)\right\}$

provided $\mu=\mu(\epsilon)>0$ is sufficiently small.

As stated in Lemma 4.1 a homogeneous Young measure $v=A v(D(x /|x|))_{x \in \Omega}$ can be generated by the gradients of a sequence $u_{k} \in \operatorname{Id}+W_{0}^{1, n-\epsilon}\left(\Omega ; \mathbb{R}^{m}\right)$ with the property of convergence in energy, even with

$$
J\left(u_{k}\right)=\langle L, v\rangle \text { meas } \Omega, k \in \mathbb{N} .
$$

However since $\langle L ; v\rangle<L^{q c}(\mathrm{Id})$ homogeneous Young measure $v$ is not a homogeneous $L$-gradient Young measure. Because of this strict inequality we also obtain that lower semicontinuity fails at Id since $\lim _{k \rightarrow \infty} J\left(u_{k}\right)<J$ (Id). Ball and Murat suggested the more general notion of $W^{1, p}$-quasiconvexity instead of Morrey's quasiconvexity as a necessary one for lower semicontinuity (in this case in the definition of quasiconvexity test functions $\phi$ should be taken in the class $\left.W_{0}^{1, p}\left(\Omega ; \mathbb{R}^{m}\right)\right)$.

However, our point of view is that all known examples of Lavrentiev phenomenon exhibit discontinuity phenomenon for deformations; this is the case when the integral functional is only a part of the total energy when we are studing the cases when the functional (1.1) presents the total energy. Therefore, at the moment we do not want to address the cases when quasiconvexity is not sufficient for lower semicontinuity, i.e. when Young measures $v_{x}$ are not homogeneous $L\left(x, u_{0}(x), \cdot\right)$ gradient Young measures. To develop a theory for such ill-posed cases we need more examples indicating the necessity to do this. In particular in [37] we even conjectured that in the case of strong materials (1.4) quasiconvexity is always sufficient for lower semicontinuity. To be more pricese we state two conjectures about strong materials.

Conjecture 1. Let $L: \mathbb{R}^{m \times n} \rightarrow \mathbb{R}$ be continuous and let $L(v) \geq \alpha|v|^{n+\epsilon}+\beta$, $\alpha>0, \epsilon>0$. Then there are no Lavrentiev phenomenon for problems with linear boundary conditions. In particular quasiconvexity and $W^{1, n+\epsilon}$-quasiconvexity are equivalent.

Conjecture 2. Let $L: \mathbb{R}^{m \times n} \rightarrow \mathbb{R}$ be a continuous quasiconvex integrand and let $L(v) \geq \alpha|v|^{n+\epsilon}+\beta, \alpha>0, \epsilon>0$. Then the associated integral functional $J$ is lower semicontinuous with respect to the weak convergence of sequences in $W^{1, n+\epsilon}$.

We have to acknowledge that the Conjectures 1 and 2 are rather brave and there are not much results indicating their validity, maybe except for the results of the paper [37]. Moreover in the case of arbitrary boundary data Foss, Hrusa and Mizel [22] suggested strong materials which admits the Lavrentiev phenomenon 
for special boundary data though they are not linear which is crucial in our conjectures. Conjectures 1 and 2 aim to clarify physical relevance of quasiconvexity and connection with the Lavrentiev phenomenon.

However in case one requires that for a.e. $x \in \Omega$ the measures $v_{x}$ is generated by the gradients of functions $\phi_{k} \in D u(x)+W_{0}^{1, p}\left(\Omega ; \mathbb{R}^{m}\right)$ with the property of convergence in energy $W^{1, p}$-quasiconvexity implies lower semicontinuity as shown recently in [30] by Mandallena.

Note that the assumption 1) implies that the Young measure $\left(v_{x}\right)_{x \in \Omega}$ satisfies the conditions (h1)-(h3) of Theorem 1.10 since conditions (h1), (h3) automatically hold because of equiboundedness of $J\left(u_{k}\right), k \in \mathbb{N}$.

Next condition on $L$-gradient Young measures is the following.

2) Let $\tilde{\Omega}$ be a compact subset of $\Omega$ such that $L: \tilde{\Omega} \times \mathbb{R}^{m} \times \mathbb{R}^{m \times n} \rightarrow \mathbb{R}$ is continuous. Let $\left(x_{k}, u_{k}\right) \in \tilde{\Omega} \times \mathbb{R}^{m}, k \in \mathbb{N}$, be such that $\left(x_{k}, u_{k}\right) \rightarrow(x, u)$ as $k \rightarrow \infty$. Assume also that $v_{k}$ are homogeneous $L\left(x_{k}, u_{k}, \cdot\right)$-gradient Young measures such that $v_{k} \rightarrow^{*} v, k \rightarrow \infty$, and $\sup _{k \in \mathbb{N}}\left\langle L\left(x_{k}, u_{k}, \cdot\right) ; v_{k}\right\rangle \leq c<\infty$. Then $v$ is a homogeneous $L(x, u, \cdot)$-gradient Young measure.

Note that in the homogeneous case $L=L(D u)$ this condition is a consequence of condition 1).

The last requirement is a possibility to approximate in strong norm and in energy functions with finite energy by more regular ones.

3) Let $u \in W^{1,1}\left(\Omega ; \mathbb{R}^{m}\right)$ with $J(u)<\infty$. Then there exists an almost piecewise affine sequence $u_{k} \in W^{1,1}\left(\Omega ; \mathbb{R}^{m}\right), k \in \mathbb{N}$, such that $u_{k} \rightarrow u$ in $W^{1,1}$ and $J\left(u_{k}\right) \rightarrow J(u)$ as $k \rightarrow \infty$.

The main result of this paper is the following theorem.

Theorem 2.6. Let $L(x, u, v): \Omega \times \mathbb{R}^{m} \times \mathbb{R}^{m \times n} \rightarrow \mathbb{R}$ be a Carathéodory integrand which is bounded on compact sets and has superlinear growth in $v \in \mathbb{R}^{m \times n}$. If assumptions 1)-3) are valid then Statements 2.1-2.4 hold. If $f \in W^{1,1}$ with $J(f)<$ $\infty$ and the approximation property 3 ) holds for $u_{k} \in W^{1,1}$, with $k \in \mathbb{N}$, such that $\left.u_{k}\right|_{\partial \Omega}=\left.f\right|_{\partial \Omega}$ for all admissible functions $u \in W^{1,1}\left(\Omega ; \mathbb{R}^{m}\right)$ then Statement 2.5 is also valid.

Theorem 2.6 suggests an efficient way to establish validity of the weak convergence theory via clarifying properties 1), 2) of Young measures generated by the gradients of functions bounded in energy and the approximation property 3). This is what we consider to be the method of gradient Young measures in the weak convergence theory. In all cases mentioned in the previous section for which weak convergence theory was established these properties hold and now we can apply Theorem 2.6 to establish it for new classes of functionals. The first such case is a case of strong materials with $p(x)$-growth, i.e. when $n+\epsilon \leq p(\cdot) \leq p<\infty$, $\epsilon>0$. 
Theorem 2.7. Let $L(x, u, v): \Omega \times \mathbb{R}^{m} \times \mathbb{R}^{m \times n} \rightarrow \mathbb{R}$ be a Carathéodory integrand with $p(x)$-growth (1.6), where $n+\epsilon \leq p(\cdot) \leq p<\infty, \epsilon>0$.

Then all the conditions 1)-3) hold, the condition 3) holds with $\left.u_{k}\right|_{\partial \Omega}=\left.u\right|_{\partial \Omega}$. Consequently Statements 2.1-2.5 are valid due to Theorem 2.6.

Another interesting class of functionals recently studied in the literature is a class of integral functionals with growth $|v|^{p}+a(x)|v|^{q}$, where $q \geq p>1$, and $a(\cdot) \geq 0$ is a continuous function. These functionals present a number of interesting properties, see $[21,31]$. It turns out that the weak convergence theory holds for strong materials with such growth.

Theorem 2.8. Let $L: \Omega \times \mathbb{R}^{m} \times \mathbb{R}^{m \times n} \rightarrow \mathbb{R}$ be a Carathéodory integrand such that

$$
\begin{aligned}
c_{1}\left(|v|^{p}+a(x)|v|^{q}\right)+c_{2} \leq L(x, u, v) & \leq c_{3}\left(|v|^{p}+a(x)|v|^{q}\right)+c_{4}, c_{3} \geq c_{1}>0, \\
q & \geq p>n+\epsilon, \epsilon>0, a(\cdot) \in C(\Omega) .
\end{aligned}
$$

Then all the conditions 1)-3) hold, the condition 3) holds with $\left.u_{k}\right|_{\partial \Omega}=\left.u\right|_{\partial \Omega}$. Consequently Statements 2.1-2.5 are valid for this class of integrands.

We prove Theorem 2.6 in Section 5 and Theorems 2.7, 2.8 in Section 6. Theorems 2.6, 2.7 were first announced in the notes $[45,46]$.

\section{Young measures as measurable functions $v: \Omega \rightarrow\left(M_{1}, \rho\right)$}

Results of this section were first stated in [36]. Complete proofs can be found in [35].

Recall a bit more general definition of weakly* convergent families of Radon measures $\left(v_{x}^{k}\right)_{x \in \Omega}$, for $k \in \mathbb{N}$, bounded in total variation by $c>0$.

Definition 3.1. A sequence $\left(v_{x}^{k}\right)_{x \in \Omega}$, for $k \in \mathbb{N}$, of Radon measures $v_{x}^{k} \in C_{0}\left(\mathbb{R}^{l}\right)^{\prime}$, where $\Omega$ is bounded and measurable subset of $\mathbb{R}^{n}$, converges weakly* to a family $\left(v_{x}\right)_{x \in \Omega}$ of Radon measures if for each $\Phi \in C_{0}\left(\mathbb{R}^{l}\right)$

$$
\left\langle\Phi ; v_{(\cdot)}^{k}\right\rangle \rightarrow^{*}\left\langle\Phi ; v_{(\cdot)}\right\rangle \text { in } L^{\infty}(\Omega), k \rightarrow \infty .
$$

A family $\left(v_{x}\right)_{x \in \Omega}$ is called homogeneous if $v_{x}=v$ for a.e. $x \in \Omega$.

The weak* convergence of the elements of the set $M_{c}\left(\mathbb{R}^{l}\right)$, which is the set of all Radon measures supported in $\mathbb{R}^{l}$ with the total variation bounded by $c$, is equivalent to convergence in the following metric

$$
\rho(\mu, \nu)=\sum_{i=1}^{\infty} \frac{1}{2^{i}\left\|\Phi_{i}\right\|_{C}} \mid\left\langle\Phi_{i} ; \mu\right\rangle-\left\langle\Phi_{i} ; \nu\right\rangle,,
$$

where $\left\{\Phi_{i}\right\} \subset C_{c}^{\infty}\left(\mathbb{R}^{l}\right)$ is a sequence dense in the space

$$
C_{0}\left(\mathbb{R}^{l}\right)=\left\{\Phi \in C\left(\mathbb{R}^{l}\right): \lim _{v \rightarrow \infty}|\Phi(v)|=0\right\} .
$$

The index " $c$ " in $C_{c}^{\infty}$ means compactness of the support. 
It is well known that $\left(M_{c}\left(\mathbb{R}^{l}\right), \rho\right)$ is a compact metric space. This follows easily from the Riesz representation theorem. If $\mu_{n} \in M_{c}\left(\mathbb{R}^{l}\right)$, then there is a subsequence $\mu_{n_{k}}$ such that the sequence $\left\langle\Phi_{i} ; \mu_{n_{k}}\right\rangle$ converges for each $i \in \mathbb{N}$. Then the functional $f: C_{0}\left(\mathbb{R}^{l}\right) \rightarrow \mathbb{R}$, which is defined at each $\Phi_{i}$ as

$$
f\left(\Phi_{i}\right):=\lim _{k \rightarrow \infty}\left\langle\Phi_{i} ; \mu_{n_{k}}\right\rangle,
$$

is a linear continuous functional bounded in norm by the constant $c$. The Riesz theorem implies $f(\cdot)=\langle\cdot ; \mu\rangle$ for a $\mu \in M_{c}\left(\mathbb{R}^{l}\right)$. Then $\rho\left(\mu_{n_{k}}, \mu\right) \rightarrow 0$ as $k \rightarrow \infty$.

The metric $\rho$ also characterizes Young measures!

Theorem 3.2. A family of probability measures $\left(v_{x}\right)_{x \in \Omega}$ is a Young measure if and only if the function $v: \Omega \rightarrow\left(M_{c}, \rho\right)$ is measurable (here $c \geq 1$ ).

Of course certain characterizations of Young measures were already in use. The next statement indicates a connection with another characterization due to Tar$\operatorname{tar}[51]$.

Proposition 3.3. Let $\Omega$ be a bounded measurable subset of $\mathbb{R}^{n}$. Let $v_{x} \in M_{c}$ for a.e. $x \in \Omega$. The family $\left(v_{x}\right)_{x \in \Omega}$ has measurable actions on elements of $C_{0}\left(\mathbb{R}^{l}\right)$ if and only if $v: \Omega \rightarrow\left(M_{c}, \rho\right)$ is a measurable mapping.

However a convenient approach turned out to be to use the characterization given by Theorem 3.2 since the measurable functions $\Omega \rightarrow\left(M_{c}, \rho\right)$ admit both general abstract properties, see Theorems 3.4, 3.5, and allow to use simple qualitative estimates given by Lemma 3.6.

Theorem 3.4. Let $\Omega \subset \mathbb{R}^{n}$ be a measurable set and let $(K, d)$ be a compact metric space. A function $\xi: \Omega \rightarrow(K, d)$ is measurable in the usual Lebesgue sense if and only if it has the Lusin property: for each $\epsilon>0$ there exists a compact subset $\Omega_{\epsilon}$ of $\Omega$ such that meas $\left(\Omega \backslash \Omega_{\epsilon}\right) \leq \epsilon$ and the function $\left.\xi\right|_{\Omega_{\epsilon}}$ is continuous.

The proof of this theorem is identical to the proof in the case in which $(K, d)$ equals $\mathbb{R}^{n}$ with the Euclidian metric.

Let $\Omega$ be a bounded measurable subset of $\mathbb{R}^{n}$ and let $(K, d)$ be a compact metric space. A mapping $V: \Omega \rightarrow 2^{K}$ is called a closed measurable multi-valued mapping if the sets $V(x) \subset K$ are closed for a.e. $x \in \Omega$ and if for each closed subset $C$ of $K$ the set $\{x \in \Omega: V(x) \cap C \neq \emptyset\}$ is measurable.

Theorem 3.5. If $V: \Omega \rightarrow 2^{K}$ is a closed measurable multivalued mapping, then there exists a measurable selection, i.e. there exists a measurable function $v: \Omega \rightarrow$ $(K, d)$ such that $v(x) \in V(x)$ for a.e. $x \in \Omega$.

This theorem was first proved in [28]. More general versions of this result can be found in [11]. 
The convergence $\left\langle\Phi ; v_{(.)}^{k}\right\rangle \rightarrow^{*}\left\langle\Phi ; v_{(\cdot)}\right\rangle$ in $L^{\infty}$ means convergence of the integrals $\int_{\tilde{\Omega}}\left\langle\Phi ; v_{x}^{k}\right\rangle d x$ to the integral $\int_{\tilde{\Omega}}\left\langle\Phi ; v_{x}\right\rangle d x$ for all measurable subsets $\tilde{\Omega}$ of $\Omega$. On the other hand the functional $\Phi \rightarrow(1 /$ meas $\tilde{\Omega}) \int_{\tilde{\Omega}}\left\langle\Phi ; v_{x}\right\rangle d x$ is the action of a Radon measure on $\Phi$ (this measure is usually denoted by $\operatorname{Av}\left(v_{x}\right)_{x \in \tilde{\Omega}}$ ):

$$
\left\langle\Phi ; \operatorname{Av}\left(v_{x}\right)_{x \in \tilde{\Omega}}\right\rangle:=\frac{1}{\operatorname{meas} \tilde{\Omega}} \int_{\tilde{\Omega}}\left\langle\Phi ; v_{x}\right\rangle d x, \forall \Phi \in C_{0}\left(\mathbb{R}^{l}\right) .
$$

To compare actions of two families of measures $\left(v_{x}^{1}\right)_{x \in \tilde{\Omega}}$ and $\left(v_{x}^{2}\right)_{x \in \tilde{\Omega}}$ we have to compare the distance between the measures $\operatorname{Av}\left(v_{x}^{1}\right)_{x \in \tilde{\Omega}}$ and $\operatorname{Av}\left(v_{x}^{2}\right)_{x \in \tilde{\Omega}}$ in $\rho$-metric.

Lemma 3.6. Let $v^{1}, v^{2}: \Omega \rightarrow\left(M_{c}, \rho\right)$ be measurable functions.

1. if $\rho\left(\operatorname{Av}\left(v_{x}^{1}\right)_{x \in \tilde{\Omega}}, \operatorname{Av}\left(v_{x}^{2}\right)_{x \in \tilde{\Omega}}\right) \leq \delta$ with $\tilde{\Omega} \subset \Omega$ such that meas $(\Omega \backslash \tilde{\Omega}) \leq$ $\delta$ meas $\Omega$, then $\rho\left(\operatorname{Av}\left(v_{x}^{1}\right)_{x \in \Omega}, \operatorname{Av}\left(v_{x}^{2}\right)_{x \in \Omega}\right) \leq(2 c+1) \delta$.

2. if $\rho\left(v_{x}^{1}, v_{x}^{2}\right) \leq \delta$ for a.e. $x \in \tilde{\Omega} \subset \Omega$ with meas $(\Omega \backslash \tilde{\Omega}) \leq \delta$ meas $\Omega$, then $\rho\left(\operatorname{Av}\left(v_{x}^{1}\right)_{x \in \Omega}, \operatorname{Av}\left(v_{x}^{2}\right)_{x \in \Omega}\right) \leq(2 c+1) \delta$.

Theorems 3.2-5 and Lemma 3.5 indicate properties of Young measures sufficient to construct the general theory and, first of all, to obtain the compactness result and the result on existence of Young measures.

Theorem 3.7 (Compactness). Let $\Omega$ be a measurable bounded subset of $\mathbb{R}^{n}$ and let $v^{k}: \Omega \rightarrow\left(M_{c}, \rho\right)$, for $k \in \mathbb{N}$, be measurable. Then there exists a subsequence (not relabeled) and a function $v: \Omega \rightarrow\left(M_{c}, \rho\right)$ such that $\left\langle\Phi ; v_{(\cdot)}^{k}\right\rangle \rightarrow^{*}$ $\left\langle\Phi ; v_{(\cdot)}\right\rangle$ in $L^{\infty}(\Omega), k \rightarrow \infty$, for each $\Phi \in C_{0}\left(\mathbb{R}^{l}\right)$.

Theorem 3.8 (Existence). In case $\left(v_{x}^{k}\right)_{x \in \Omega}$, for $k \in \mathbb{N}$, is a sequence of Young measures with the property $\int_{\Omega}\left\langle L ; v_{x}^{k}\right\rangle d x \leq c<\infty, k \in \mathbb{N}$, where $L(v) \rightarrow \infty$ as $|v| \rightarrow \infty$, there exists a subsequence $\left(v_{x}^{k}\right)_{x \in \Omega}, k \in \mathbb{N}$, (not relabeled) and a Young measure $\left(v_{x}\right)_{x \in \Omega}$ with the property $\left(v_{x}^{k}\right)_{x \in \Omega} \rightarrow^{*}\left(v_{x}\right)_{x \in \Omega}, k \rightarrow \infty$.

Theorem 3.8 is a generalization of Theorem 1.2.

Theorem 3.9 (Lower Semicontinuity). Let $\Omega$ be a bounded measurable subset of $\mathbb{R}^{n}$ and let $L: \Omega \times \mathbb{R}^{l} \rightarrow \overline{\mathbb{R}}$ be a Carathéodory integrand bounded from below. Let also $\left(v_{x}^{k}\right)_{x \in \Omega}$, for $k \in \mathbb{N}$, be a sequence of Young measures that converges weakly* to a Young measure $\left(v_{x}\right)_{x \in \Omega}$.

Then

$$
\liminf _{k \rightarrow \infty} \int_{\Omega}\left\langle L(x, \cdot) ; v_{x}^{k}\right\rangle d x \geq \int_{\Omega}\left\langle L(x, \cdot) ; v_{x}\right\rangle d x .
$$

We will frequently use also the following two standard results.

Lemma 3.10. Consider a bounded measurable subset $\Omega$ of $\mathbb{R}^{n}$ and suppose that a sequence $\xi_{k}: \Omega \rightarrow \mathbb{R}^{l}$ generates a Young measure $\left(v_{x}\right)_{x \in \Omega}$. Then $\xi_{k}$ converges in measure if and only if $\nu_{x}=\delta_{\xi(x)}$ a.e. in $\Omega$. 
Lemma 3.11. Let $L_{k}: \mathbb{R}^{m \times n} \rightarrow \mathbb{R}$ be continuous integrands such that $L_{k} \geq$ const, $k \in \mathbb{N}$, and assume that $L_{k} \rightarrow L$ uniformly on compact sets as $k \rightarrow \infty$. Assume also that for $v_{k}, k \in \mathbb{N}$, are probability measures supported in $\mathbb{R}^{m \times n}$ and that $v_{k} \rightarrow^{*} v$, as $k \rightarrow \infty$, where $v$ is also a probability measure. Then

$$
\liminf _{k \rightarrow \infty}\left\langle L_{k} ; v_{k}\right\rangle \geq\langle L ; v\rangle .
$$

If $v$ is a probability measure supported in $\mathbb{R}^{l}$ and centered at $A \in \mathbb{R}^{l}$ we say that a probability measure $v \diamond \tilde{A}$ is obtained by shifting the center of mass to $\tilde{A}$ whenever

$$
\langle\Phi ; v \diamond \tilde{A}\rangle=\langle\Phi(\cdot-A+\tilde{A}) ; v\rangle, \forall \Phi \in C_{0}\left(\mathbb{R}^{l}\right) .
$$

It easy to see that $\rho\left(\nu \diamond A_{k}, v \diamond \tilde{A}\right) \rightarrow 0$ if $A_{k} \rightarrow \tilde{A}$ as $k \rightarrow \infty$ due to equi-continuity of the functions $\Phi_{i}, i \in \mathbb{N}$, defining the metric $\rho$.

\section{Proof of Theorem 1.10}

In this section we prove Theorem 1.10.

First we show how to construct a typical $L$-gradient (homogeneous $L$-gradient) Young measure. Let $L: \mathbb{R}^{m \times n} \rightarrow \overline{\mathbb{R}}$ be a continuous integrand with at least linear growth. Let $\phi \in W_{0}^{1,1}\left(\Omega ; \mathbb{R}^{m}\right)$ and let $A \in \mathbb{R}^{m \times n}$. Then $A v(A+D \phi)_{\Omega}$ is a probability measure defined by

$$
\left\langle\Phi ; A v(A+D \phi)_{\Omega}\right\rangle:=\frac{1}{\operatorname{meas} \Omega} \int_{\Omega} \Phi(A+D \phi(x)) d x, \forall \Phi \in C_{0}\left(\mathbb{R}^{m \times n}\right) .
$$

Lemma 4.1. The measure $A v(A+D \phi)_{\Omega}$ is an L-gradient Young measure and

$$
\int_{\Omega} L(A+D \phi(x)) d x=\left\langle L ; A v(A+D \phi)_{\Omega}\right\rangle \text { meas } \Omega
$$

for any continuous integrand $L: \mathbb{R}^{m \times n} \rightarrow \overline{\mathbb{R}}$ and any $A \in \mathbb{R}^{m \times n}$ provided $\int_{\Omega} L(A+D \phi(x)) d x<\infty$.

If $\phi \in W_{0}^{1, \infty}\left(\Omega ; \mathbb{R}^{m}\right)$ then the measure $A v(A+D \phi)_{\Omega}$ is a homogeneous $L$ gradient Young measure.

A proof of this lemma follows from a folklore result widely used in literature, but, seemingly, its first author was Bogolubov, see [7].

Proposition 4.2. Consider open bounded subsets $\Omega$ and $\tilde{\Omega}$ of $\mathbb{R}^{n}$ with Lipschitz boundary. Take $\phi \in W_{0}^{1, p}\left(\Omega ; \mathbb{R}^{m}\right)$ with $1 \leq p \leq \infty$. Given $\epsilon>0$ consider a decomposition of $\tilde{\Omega}$ into subsets of the form $x_{i}+\epsilon_{i} \Omega$ with $\epsilon_{i} \leq \epsilon, i \in \mathbb{N}$, and a measure zero set (see, e.g., [49, page 109]). Define $\tilde{\phi}: \tilde{\Omega} \rightarrow \mathbb{R}^{m}$ as follows:

$$
\tilde{\phi}= \begin{cases}\epsilon_{i} u\left(\left(x-x_{i}\right) / \epsilon_{i}\right) & \text { for } x \in\left(x_{i}+\epsilon_{i} \Omega\right) \text { and } i \in \mathbb{N} \\ 0 & \text { otherwise. }\end{cases}
$$


Then $\tilde{\phi} \in W_{0}^{1, p}\left(\tilde{\Omega} ; \mathbb{R}^{m}\right)$ and for every bounded from below continuous function $L: \mathbb{R}^{m \times n} \rightarrow \overline{\mathbb{R}}$ and every $A \in \mathbb{R}^{m \times n}$ we have

$$
\begin{aligned}
\frac{1}{\operatorname{meas} \Omega} \int_{\Omega} L(A+D \phi(x)) d x & =\frac{1}{\operatorname{meas} \tilde{\Omega}} \int_{\tilde{\Omega}} L(A+D \tilde{\phi}(x)) d x, \\
\frac{\|\tilde{\phi}\|_{L^{p}}}{\text { meas } \tilde{\Omega}} & \leq \frac{\epsilon \phi \|_{L^{p}}}{\text { meas } \Omega} .
\end{aligned}
$$

Proof of Lemma 4.1. Take the functions $\tilde{\phi}_{k}$ in Proposition 4.2 with $\epsilon \leq 1 / k, k \in \mathbb{N}$. Assume also that for each $k \in \mathbb{N}$ and $k^{\prime} \geq k$ we have that if $x_{i_{k^{\prime}}}+\epsilon_{i_{k^{\prime}}} \Omega$ is an element of the decomposition associated with $k^{\prime}$ then $x_{i_{k^{\prime}}}+\epsilon_{i_{k^{\prime}}} \Omega \subset x_{i_{k}}+\epsilon_{i_{k}} \Omega$ for certain $i_{k}$. Then

$$
A v\left(A+D \tilde{\phi}_{k^{\prime}}\right)_{x_{i_{k}}+\epsilon_{i_{k}} \Omega}=A v\left(A+D \tilde{\phi}_{k}\right)_{x_{i_{k}}+\epsilon_{i_{k}} \Omega}=A v(A+D \phi)_{\Omega}, k^{\prime} \geq k
$$

Verify that $\left(A+D \tilde{\phi}_{k}\right)$ generates the measure $A v(A+D \phi)_{\Omega}$.

By Theorem 3.7 a subsequence $A+D \tilde{\phi}_{k_{l}}$ generate a Young measure $\left(v_{x}\right)_{x \in \Omega}$. Take a Lebesgue point $x_{0}$ of $\left(v_{x}\right)_{x \in \Omega}$ and suppose $x_{0} \in x_{i_{l}}+\epsilon_{i_{l}} \Omega$ with suitable $i_{l} \in \mathbb{N}$. Then Proposition 4.2 yields

$$
A v\left(A+D \tilde{\phi}_{l^{\prime}}\right)_{x_{i_{l}}+\epsilon_{i_{l}} \Omega}=A v(A+D \phi)_{\Omega}, \text { for } l \in \mathbb{N}, \text { and } l^{\prime} \geq l .
$$

On the other hand

$$
A v\left(A+D \tilde{\phi}_{i_{l}^{\prime}}\right)_{x_{i^{\prime}}}+\epsilon_{i_{l}} \Omega \rightarrow^{*} A v\left(v_{x}\right)_{x_{i_{l}}+\epsilon_{i l} \Omega}, \text { as } l^{\prime} \rightarrow \infty
$$

and

$$
A v\left(v_{x}\right)_{x_{i_{l}}+\epsilon_{i_{l}} \Omega} \rightarrow^{*} v_{x_{0}} \text {, as } i \rightarrow \infty,
$$

see Lemma 3.6. Therefore $v_{x_{0}}=A v(A+D \phi)_{\Omega}$. Then the Young measure $\left(v_{x}\right)_{x \in \Omega}$ is homogeneous and equal to $A v(A+D \phi)_{\Omega}$ a.e.: it is also an $L$-gradient Young measure since

$$
\int_{\tilde{\Omega}} L\left(A+D \tilde{\phi}_{k}(x)\right) d x=\left\langle L ; A v(A+D \phi)_{\Omega}\right\rangle \text { meas } \tilde{\Omega} \text {, for } k \in \mathbb{N} .
$$

Since every subsequence of $\tilde{\phi}_{k}$ includes a subsequence whose gradients generate $A v(A+D \phi)_{\Omega}$, the gradients of the original sequence $\tilde{\phi}_{k}$ also generate this measure.

Remark 4.3. Note that $A v(A+D \tilde{\phi})_{\tilde{\Omega}}$ is also $L$-gradient (homogeneous $L$-gradient in the case $\left.\phi \in W_{0}^{1, \infty}\left(\Omega ; \mathbb{R}^{m}\right)\right)$ Young measure for any bounded open subset $\tilde{\Omega}$ of $\mathbb{R}^{n}$ and

$$
A v(A+D \phi)_{\Omega}=A v(A+D \tilde{\phi})_{\tilde{\Omega}}
$$

for functions $\phi, \tilde{\phi}$ from Proposition 4.2. 
We will need also the following lemma.

Lemma 4.4. Let $L(x, u, v): \Omega \times \mathbb{R}^{m} \times \mathbb{R}^{m \times n} \rightarrow \overline{\mathbb{R}}$ be a Carathéodory integrand with at least linear growth in $v$ and let $\left(v_{x}^{k}\right)_{x \in \Omega}$, for $k \in \mathbb{N}$, be L-gradient Young measures such that the centers of mass of $\nu_{x}^{k}$ are $D u_{k}(x)$ for a.e. $x \in \Omega$, and $k \in \mathbb{N}$. Assume

$$
\left(v_{x}^{k}\right)_{x \in \Omega} \rightarrow^{*}\left(v_{x}\right)_{x \in \Omega}, \quad u_{k} \rightarrow u_{0} \in W^{1,1}\left(\Omega ; \mathbb{R}^{m}\right) .
$$

Assume also that

$$
\int\left\langle L\left(x, u_{k}(x), \cdot\right) ; v_{x}^{k}\right\rangle d x \rightarrow \int_{\Omega}\left\langle L\left(x, u_{0}(x), \cdot\right) ; v_{x}\right\rangle<\infty .
$$

Then $\left(v_{x}\right)_{x \in \Omega}$ is also an L-gradient Young measure and the centers of mass of $v_{x}$ are $D u_{0}(x)$ a.e. in $\Omega$.

The proof follows from the standard diagonalization arguments and we leave it to the reader.

Now we are in position to prove Theorem 1.10.

Proof of Theorem 1.10. For each $M \in \mathbb{N}$ we can find a compact subset $\Omega_{M}$ of $\Omega$ with meas $\left(\Omega \backslash \Omega_{M}\right) \leq 1 / M$ such that the restrictions of $u$, and $D u$ to $\Omega_{M}$ are continuous, the restriction of $L$ to $\Omega_{M} \times \mathbb{R}^{m} \times \mathbb{R}^{m \times n}$ is continuous, the restriction of $v_{(\cdot)}$ to $\Omega_{M}$ is continuous in $\rho$-metric and finally the function $\left\langle L(x, u(x), \cdot) ; v_{x}\right\rangle$ : $\Omega_{M} \rightarrow \mathbb{R}$ is also continuous. We can also assume that for each $x \in \Omega_{M}$ the measure $v_{x}$ is a homogeneous $L(x, u(x), \cdot)$-gradient Young measure.

Let $x_{0} \in \Omega_{M}$ be a Lebesgue point of $\Omega_{M}$. Since $v_{x_{0}}$ is a homogeneous $L\left(x_{0}, u\left(x_{0}\right), \cdot\right)$-gradient Young measure, there exists a sequence $\phi_{i} \in W_{0}^{1, \infty}\left(\Omega ; \mathbb{R}^{m}\right)$ such that $\left(D u\left(x_{0}\right)+D \phi_{i}\right)_{x \in \Omega}$ generates $v_{x_{0}}$ as a homogeneous $L\left(x_{0}, u\left(x_{0}\right), \cdot\right)$-gradient Young measure. Then

$$
\begin{gathered}
\rho\left(A v\left(D u\left(x_{0}\right)+D \phi_{i}\right)_{\Omega} ; v_{x_{0}}\right) \rightarrow 0 \\
\left\langle L\left(x_{0}, u\left(x_{0}\right), \cdot\right) ; A v\left(D u\left(x_{0}\right)+D \phi_{i}\right)_{\Omega}\right\rangle \rightarrow\left\langle L\left(x_{0}, u\left(x_{0}\right), \cdot\right) ; v_{x_{0}}\right\rangle, \text { as } i \rightarrow \infty .
\end{gathered}
$$

We can find an $\epsilon_{0}>0$ and $i_{0}=i\left(x_{0}\right)$ such that

$$
\begin{gathered}
\rho\left(A v\left(D u(x)+D \phi_{i_{0}}\right)_{\Omega} ; v_{x}\right) \leq 1 / M, \\
\left|\left\langle L(x, u(x), \cdot) ; A v\left(D u(x)+D \phi_{i_{0}}\right)_{\Omega}\right)\right\rangle-\left\langle L(x, u(x), \cdot) ; v_{x}\right\rangle \mid<1 / M
\end{gathered}
$$

for $x \in\left(B\left(x_{0}, \epsilon_{0}\right) \cap \Omega_{M}\right)$. This follows from the continuity assumptions.

We can then isolate a finite collection of disjoint balls $B\left(x_{j}, \epsilon_{j}\right)$, for $j \in$ $\{1, \ldots, j(M)\}$ such that (4.2) holds in each ball $B\left(x_{j}, \epsilon_{j}\right)$ with certain $\phi_{j} \in W_{0}^{1, \infty}\left(\Omega ; \mathbb{R}^{m}\right)$ instead of $\phi_{i_{0}}$. Assume also that

$$
\operatorname{meas}\left(\Omega_{M} \backslash \cup_{j=1}^{j(M)} B\left(x_{j}, \epsilon_{j}\right)\right) \leq 1 / M .
$$


Define a Young measure $\left(v_{x}^{M}\right)_{x \in \Omega}$ as follows

$$
\begin{cases}v_{x}^{M}=A v\left(D u(x)+D \phi_{j}\right)_{\Omega}, & \text { for } x \in B\left(x_{j}, \epsilon_{j}\right) \cap \Omega_{M} \\ v_{x}^{M}=\delta_{D u(x)} & \text { and } j \in\{1, \ldots, j(M)\} \\ \text { otherwise. }\end{cases}
$$

Note that

$$
\rho\left(v_{x}^{M} ; v_{x}\right)<1 / M, \text { for } x \in \cup_{j}\left(B\left(x_{j}, \epsilon_{j}\right) \cap \Omega_{M}\right) .
$$

Then (4.3), (4.5) imply that by Lemma 3.6

$$
\left(v_{x}^{M}\right)_{x \in \Omega} \rightarrow^{*}\left(v_{x}\right)_{x \in \Omega}, \text { as } M \rightarrow \infty .
$$

The inequalities (4.2) also imply that

$$
\int_{\Omega}\left\langle L(x, u(x), \cdot) ; v_{x}^{M}\right\rangle d x \rightarrow \int_{\Omega}\left\langle L(x, u(x), \cdot) ; v_{x}\right\rangle d x, \text { as } M \rightarrow \infty .
$$

Then $\left(v_{x}\right)_{x \in \Omega}$ is an $L$-gradient Young measure provided $\left(v_{x}^{M}\right)_{x \in \Omega}$, for $M \in \mathbb{N}$, are, see Lemma 4.4.

Therefore we only need to show that for each $M \in \mathbb{N}$ the Young measure $\left(v_{x}^{M}\right)_{x \in \Omega}$ is an $L$-gradient Young measure. erties

Let $u_{k} \in W^{1,1}\left(\Omega ; \mathbb{R}^{m}\right)$ be an almost piece-wise affine sequence with the prop-

$$
u_{k} \rightarrow u \text { in } W^{1,1}, L\left(\cdot, u_{k}(\cdot), D u_{k}(\cdot)\right) \rightarrow L(\cdot, u(\cdot), D u(\cdot)) \text { in } L^{1}
$$

and let $\Omega_{k} \subset \Omega$ be open subsets of $\Omega$ such that the restrictions of $u_{k}$ to $\Omega_{k}$ are piece-wise affine. Then there exist compact sets $\tilde{\Omega}_{k} \subset \Omega_{k}$ such that

$$
\begin{gathered}
\left\|u_{k}-u\right\|_{L^{\infty}\left(\tilde{\Omega}_{k}\right)} \rightarrow 0,\left\|D u_{k}-D u\right\|_{L^{\infty}\left(\tilde{\Omega}_{k}\right)} \rightarrow 0, \text { as } k \rightarrow \infty \\
\operatorname{meas}\left(\Omega_{M} \backslash \tilde{\Omega}_{k}\right) \rightarrow 0 .
\end{gathered}
$$

For each $j \in\{1, \ldots, j(M)\}$ consider the set $B\left(x_{j}, \epsilon_{j}\right) \cap \tilde{\Omega}_{k}$ and define

$$
\begin{gathered}
v_{x}^{M, k}=A v\left(D u_{k}(x)+D \phi_{j}\right)_{\Omega}, \text { for } x \in B\left(x_{j}, \epsilon_{j}\right) \cap \tilde{\Omega}_{k} \cap \Omega_{M}, \text { and } j \in\{1, \ldots, j(M)\}, \\
v_{x}^{M, k}=\delta_{D u_{k}(x)}, \text { for } x \in \Omega \backslash \cup_{j=1}^{j(M)}\left(B\left(x_{j}, \epsilon_{j}\right) \cap \tilde{\Omega}_{k} \cap \Omega_{M}\right) .
\end{gathered}
$$

Then the convergences (4.9) imply

$$
\left(v_{x}^{M, k}\right)_{x \in \Omega} \rightarrow^{*}\left(v_{x}^{M}\right)_{x \in \Omega}, k \rightarrow \infty .
$$

Due to compactness of the supports of $A v\left(D u\left(x_{j}\right)+D \phi_{j}\right)_{\Omega}, j \in\{1, \ldots, j(M)\}$, and boundedness of $L$ on compact sets we also obtain

$$
\int_{\Omega}\left\langle L\left(x, u_{k}(x), \cdot\right) ; v_{x}^{M, k}\right\rangle d x \rightarrow \int_{\Omega}\left\langle L(x, u(x), \cdot) ; v^{M}\right\rangle d x, k \rightarrow \infty .
$$


Lemma 4.4 implies that $\left(v_{x}^{M}\right)_{x \in \Omega}$ is an $L$-gradient Young measure provided $\left(v_{x}^{M, k}\right)_{x \in \Omega}$ are. We have to show the latter. Note that without loss of generality we can assume that given $k \in \mathbb{N}$ the set $\Omega_{k}$ can be decomposed into finite collection of open sets $\Omega_{k, i}, i \in\{1, \ldots, i(k)\}$ where $u_{k}$ is affine, i.e.

$$
D u_{k}(x)=A_{k, i}, x \in \Omega_{k, i}, i \in\{1, \ldots, i(k)\} .
$$

It remains to be shown that in each set $\Omega_{k, i} \cap B\left(x_{j}, \epsilon_{j}\right)$, and $j \in\{1, \ldots, j(M)\}$, and $i \in\{1, \ldots, i(k)\}$ the Young measure $\left(v_{x}^{M, k}\right)_{x \in\left(\Omega_{k, i} \cap B\left(x_{j}, \epsilon_{j}\right)\right)}$ given by

$$
\begin{gathered}
v_{x}^{M, k}=A v\left(A_{k, i}+D \phi_{j}\right)_{\Omega}, \text { for } x \in\left(\Omega_{k, i} \cap \tilde{\Omega}_{k} \cap B\left(x_{j}, \epsilon_{j}\right)\right), \\
v_{x}^{M, k}=\delta_{D u_{k}(x)}, \text { for } x \in\left(\left(\Omega_{k, i} \cap B\left(x_{j}, \epsilon_{j}\right)\right) \backslash \tilde{\Omega}_{k}\right)
\end{gathered}
$$

is an $L$-gradient Young measure. It is easy to do the since for every $i \in\{1, \ldots, i(k)\}$, $j \in\{1, \ldots, j(M)\}$, we can select a sequence of open subsets $\Omega_{i_{l}}$, for $l \in \mathbb{N}$, of $\Omega_{k, i}$ such that $\left(\Omega_{k, i} \cap \tilde{\Omega}_{k} \cap B\left(x_{j}, \epsilon_{j}\right)\right) \subset \Omega_{i_{l}}$ and

$$
\text { meas }\left(\Omega_{i_{l}} \backslash\left(\Omega_{k, i} \cap \tilde{\Omega}_{k} \cap B\left(x_{j}, \epsilon_{j}\right)\right)\right) \rightarrow 0, \text { as } l \rightarrow \infty .
$$

Consider a Young measure $\left(v_{x}^{i_{l}}\right)_{x \in \Omega_{k, i} \cap B\left(x_{j}, \epsilon_{j}\right)}$ such that

$$
\begin{aligned}
& v_{x}^{i_{l}}=A v\left(A_{k, i}+D \phi_{j}\right)_{\Omega}, \text { for } x \in \Omega_{i_{l}}, \\
& v_{x}^{i_{l}}=\delta_{A_{k, i}}, \quad x \in\left(\left(\Omega_{k, i} \cap B\left(x_{j}, \epsilon_{j}\right)\right) \backslash \Omega_{i_{l}}\right) .
\end{aligned}
$$

Then

$$
\begin{gathered}
\left(v_{x}^{i_{l}}\right)_{x \in\left(\Omega_{k, i} \cap B\left(x_{j}, \epsilon_{j}\right)\right)} \rightarrow^{*}\left(v_{x}^{M, k}\right)_{x \in\left(\Omega_{k, i} \cap B\left(x_{j}, \epsilon_{j}\right)\right)} \text {, as } l \rightarrow \infty, \\
\int_{\Omega_{k, i} \cap B\left(x_{j}, \epsilon_{j}\right)}\left\langle L\left(x, u_{k}(x), \cdot\right) ; v_{x}^{i_{l}}\right\rangle d x \rightarrow \int_{\Omega_{k, i} \cap B\left(x_{j}, \epsilon_{j}\right)}\left\langle L\left(x, u_{k}(x), \cdot\right) ; v_{x}^{M, k}\right\rangle d x, \text { as } l \rightarrow \infty .
\end{gathered}
$$

Then Lemma 4.4 implies that $\left(v_{x}^{M, k}\right)_{x \in \Omega_{k, i}}$ is an $L$-gradient Young measure since $\left(v_{x}^{i_{l}}\right)_{x \in\left(\Omega_{k, i} \cap B\left(x_{j}, \epsilon_{j}\right)\right)}$ obviously are. Then $\left(v_{x}^{M, k}\right)_{x \in \Omega}$ is an $L$-gradient Young measure, which proves the theorem in the vectorial case.

Now we have to prove Theorem 1.10 in the remaining scalar case $(m=1)$ when we allow the integrand to be extended-valued. In the scalar case given a continuous integrand $L: \mathbb{R}^{n} \rightarrow \overline{\mathbb{R}}$ with at least linear growth any probability measure with finite action on $L$ is a homogeneous $L$-gradient Young measure, see [38]. It can be approximated in $\rho$-metric and in energy by convex combinations of Dirac masses $\sum_{i=1}^{i(k)} c_{i_{k}} \delta_{v_{i_{k}}}$, for $k \in \mathbb{N}$, with the same center of mass. On the other hand given a convex combination $v=\sum_{i=1}^{q} c_{i} \delta_{v_{i}}$ of Dirac masses centered at $A$ we can find a sequence of functions $\phi_{j} \in l_{A}+W_{0}^{1, \infty}(\Omega)$ such that $D \phi_{j}$ generate $v$ as a homogeneous $L$-gradient Young measure and $D \phi_{j} \in \cup_{i=1}^{q} B\left(v_{i}, 1 / j\right)$, for $j \in \mathbb{N}$, a.e. in $\Omega$, see [40]. Then the same scheme as in the vectorial case can be applied to prove Theorem 1.10 when $\phi_{j}$ in definition of $\left(v_{x}^{M}\right)_{x \in \Omega}$ is selected in this way. 


\section{Proof of Theorem 2.6}

In order to prove Theorem 2.6 we will need two auxiliary lemmas.

Lemma 5.1. Let $L: \mathbb{R}^{m \times n} \rightarrow \overline{\mathbb{R}}$ be a continuous integrand bounded from below. Then $L$ is quasiconvex at $A \in \mathbb{R}^{m \times n}$ if and only if Jensen's inequality $\langle L ; v\rangle \geq$ $L(A)$ holds for each homogeneous L-gradient Young measure $v$ centered at $A$.

We also have

$L^{q c}(A)=\inf \{\langle L ; \nu\rangle: \nu$ is a homogeneous L-gradient Young measure centered at $A\}$.

Proof. In case $L$ is quasiconvex at $A \in \mathbb{R}^{m \times n}$ we have

$$
\begin{aligned}
& \int_{\Omega} L(A+D \phi(x)) d x \geq L(A) \text { meas } \Omega \\
& \qquad \text { for each } \phi \in W_{0}^{1, \infty}\left(\Omega ; \mathbb{R}^{m \times n}\right)
\end{aligned}
$$

Since for each homogeneous $L$-gradient Young measure $v$ centered at $A$ there exists a sequence $\phi_{k} \in W_{0}^{1, \infty}\left(\Omega ; \mathbb{R}^{m \times n}\right)$ with the property

$$
\int_{\Omega} L\left(A+D \phi_{k}(x)\right) d x \rightarrow\langle L ; v\rangle \text { meas } \Omega, \text { as } k \rightarrow \infty,
$$

we infer

$$
\langle L ; v\rangle \text { meas } \Omega \geq L(A) \text { meas } \Omega,
$$

i.e. Jensen's inequality $\langle L ; v\rangle \geq L(A)$ really holds.

Conversely if Jensen's inequality holds for each homogeneous $L$-gradient Young measure centered at $A$ then given $\phi \in W_{0}^{1, \infty}\left(\Omega ; \mathbb{R}^{m \times n}\right)$ the inequality also holds for measure $A v(A+D \phi)_{\Omega}$, i.e.

$$
\int_{\Omega} L(A+D \phi(x)) d x=\left\langle L ; A v(A+D \phi)_{\Omega}\right\rangle \text { meas } \Omega \geq L(A) \text { meas } \Omega
$$

which in turn quasiconvexity of $L$ at $A$. This proves the first part of the lemma.

To show that

$$
\begin{array}{r}
L^{q c}(A)=\inf \{\langle L ; v\rangle ; v \text { is a homogeneous L-gradient Young } \\
\text { measure centered at } A\}
\end{array}
$$

only notice that for each $\phi \in W_{0}^{1, \infty}\left(\Omega ; \mathbb{R}^{m \times n}\right)$ we have

$$
\int L(A+D \phi(x)) d x=\left\langle L ; A v(A+D \phi)_{\Omega}\right\rangle .
$$

This and Definition 1.5 of homogeneous $L$-gradient Young measures imply the validity of (5.1). 
Lemma 5.2. Let $L: \mathbb{R}^{m \times n} \rightarrow \overline{\mathbb{R}}$ be a continuous integrand with superlinear growth. Assume also that $L$ has property 2 ) of Section 2, i.e. given homogeneous $L$-gradient Young measures $v_{k}$, as $k \in \mathbb{N}$, such that $v_{k} \rightarrow^{*} v$, as $k \rightarrow \infty$, and $\left\langle L ; v_{k}\right\rangle \leq c<\infty$, for $k \in \mathbb{N}$, then $v$ is a homogeneous L-gradient Young measure.

Furthermore $L$ is strictly quasiconvex at $A$ if and only if the strict version of Jensen's inequality $\langle L ; v\rangle>L(A)$ holds for each nontrivial homogeneous $L$ gradient Young measure $v$ centered at $A$.

Proof. It is easy to see that strict quasiconvexity at $A$ is equivalent to the following property: given $\epsilon>0$ there exists $\delta=\delta(\epsilon)>0$ such that

$$
\int_{\Omega} L(A+D \phi(x)) d x \geq(L(A)+\delta) \text { meas } \Omega
$$

for each $\phi \in W_{0}^{1, \infty}\left(\Omega ; \mathbb{R}^{m \times n}\right)$ with the property meas $\{x \in \Omega:|D \phi(x)| \geq \epsilon\} \geq \epsilon$. If $L$ is strictly quasiconvex at $A$ and $v$ is a nontrivial homogeneous $L$-gradient Young measure centered at $A$, then there exists a sequence $\phi_{k} \in W_{0}^{1, \infty}\left(\Omega ; \mathbb{R}^{m \times n}\right)$, for $k \in \mathbb{N}$, such that $A+D \phi_{k}$ generates $v$ as a homogeneous $L$-gradient Young measure. Due to nontriviality of $v$ we have meas $\left\{x \in \Omega:\left|D \phi_{k}(x)\right| \geq \epsilon\right\} \geq \epsilon$ for some $\epsilon>0, k \in \mathbb{N}$. Since

$$
\int_{\Omega} L\left(A+D \phi_{k}(x)\right) d x \rightarrow\langle L ; v\rangle \text { meas } \Omega, \text { as } k \rightarrow \infty,
$$

we obtain

$$
\langle L ; v\rangle \geq(L(A)+\delta),
$$

i.e. the strict version of Jensen's inequality holds.

To show the converse we notice that if $\phi_{k} \in W_{0}^{1, \infty}\left(\Omega ; \mathbb{R}^{m}\right)$ with the property meas $\left\{x \in \Omega:\left|D \phi_{k}(x)\right| \geq \epsilon\right\} \geq \epsilon$ then for a subsequence (not relabeled) we have that $A v\left(A+D \phi_{k}\right)_{\Omega} \rightarrow^{*} v$, as $k \rightarrow \infty$, and $v$ is a nontrivial probability measure. By property 2) of Section $2 v$ is a homogeneous $L$-gradient Young measure. Since we have by Theorem 1.3

$$
\liminf _{k \rightarrow \infty} J\left(l_{A}+\phi_{k}\right) \geq\langle L ; v\rangle \text { meas } \Omega
$$

and since we also have the strict version of Jensen's inequality $\langle L ; v\rangle>L(A)$ the validity of strict quasiconvexity at $A$ follows.

Proof of Theorem 2.6. Assume that the properties 1-3) stated in Section 2 are valid. We have to show then the validity of Statements 2.1, 2.2, 2.4, 2.5.

\section{1) Lower semicontinuity (Statement 2.1)}

Let $u_{k} \in W^{1,1}\left(\Omega ; \mathbb{R}^{m}\right)$, for $k \in \mathbb{N}$, and assume that $u_{k} \rightarrow u$ in $W^{1,1}$ and that $\liminf _{k \rightarrow \infty} J\left(u_{k}\right)<\infty$. Then we can assume that $D u_{k}$ generate a Young measure $\left(v_{x}\right)_{x \in \Omega}$. By Theorem 1.3 we have

$$
\liminf _{k \rightarrow \infty} J\left(u_{k}\right) \geq \int_{\Omega}\left\langle L(x, u(x), \cdot) ; v_{x}\right\rangle d x
$$


and for a.e. $x \in \Omega$ measure $v_{x}$ is a homogeneous $L(x, u(x), \cdot)$-gradient Young measure, $c f$. property 1$)$ of Section 2 . In case $L(x, u(x), \cdot)$ is quasiconvex at $D u(x)$ Lemma 5.1 implies

$$
\left\langle L(x, u(x), \cdot) ; v_{x}\right\rangle \geq L(x, u(x), D u(x)) .
$$

The inequalities (5.1), (5.2) imply that

$$
\liminf _{k \rightarrow \infty} J\left(u_{k}\right) \geq J(u)
$$

i.e. lower semicontinuity really holds.

Assume now that lower semicontinuity holds at $u \in W^{1,1}$. We have to show that $L(x, u(x), \cdot)$ is quasiconvex at $D u(x)$ for a.e. $x \in \Omega$. Let $\Omega_{M} \subset \Omega$ be a compact set such that $u: \Omega_{M} \rightarrow \mathbb{R}^{m}, D u: \Omega_{M} \rightarrow \mathbb{R}^{m \times n}$ are continuous and also $L: \Omega_{M} \times \mathbb{R}^{m} \times \mathbb{R}^{m \times n} \rightarrow \mathbb{R}$ is continuous. Let $x$ be a Lebesgue point of $\Omega_{M}$. In case $L(x, u(x), \cdot)$ is not quasiconvex at $D u(x)$ we can use Lemma 5.1 to find a homogeneous $L(x, u(x), \cdot)$-gradient Young measure $v_{x}$ such that

$$
\left\langle L(x, u(x), \cdot) ; v_{x}\right\rangle<L(x, u(x), D u(x)) .
$$

Since $v_{x}$ is a homogeneous $L(x, u(x), \cdot)$-gradient Young measure there exists a sequence $\phi_{k} \in W_{0}^{1, \infty}\left(\Omega ; \mathbb{R}^{m}\right)$ such that $A+D \phi_{k}$ generate $v$ as a homogeneous $L(x, u(x), \cdot)$-gradient Young measure. In particular

$$
\int_{\Omega} L\left(x, u(x), A+D \phi_{k}(y)\right) d y \rightarrow\langle L ; v\rangle \text { meas } \Omega .
$$

Then for sufficiently large $k \in \mathbb{N}$ we have

$$
\left\langle L(x, u(x), \cdot) ; A v\left(A+D \phi_{k}\right)_{\Omega}\right\rangle<L(A) \text { meas } \Omega .
$$

Denote $A v\left(A+\phi_{k}\right)_{\Omega}$ by $\tilde{v}$.

For all $y \in \Omega_{M}$ sufficiently close to $x$ we can define $v_{y}$ by $\tilde{v} \diamond D u(y)$. Then

$$
\left\langle L(y, u(y), \cdot) ; v_{y}\right\rangle<L(y, u(y), D u(y))
$$

for all such $y$. Now we define $\left(\tilde{v}_{x}\right)_{x \in \Omega}$ as $v_{y}$ for such $y$ and $\tilde{v}=\delta_{D u(x)}$ otherwise. By Theorem $1.10\left(\tilde{v}_{x}\right)_{x \in \Omega}$ is an $L$-gradient Young measure, i.e. there exists a sequence $u_{k} \in W^{1,1}\left(\Omega ; \mathbb{R}^{m}\right)$ such that $u_{k} \rightarrow u$ in $W^{1,1}$ and $D u_{k}$ generates $\left(\tilde{v}_{x}\right)_{x \in \Omega}$ with the property

$$
L\left(\cdot, u_{k}(\cdot), D u_{k}(\cdot)\right) \rightarrow\left\langle L(\cdot, u(\cdot), v) ; \tilde{v}_{(\cdot)}\right\rangle \text { in } L^{1} .
$$

Then

$$
\liminf _{k \rightarrow \infty} J\left(u_{k}\right)=\int_{\Omega}\left\langle L(x, u(x), \cdot) ; v_{x}\right\rangle d x<J(u)
$$


which is a conradiction with the assumption of lower semicontinuity of $J$. Therefore $L(x, u(x), \cdot)$ should be quasiconvex at all Lebesgue points $\Omega_{M}$. Then $L(x, u(x), \cdot)$ is quasiconvex at $D u(x)$ a.e. in $\Omega$.

\section{2) Convergence in energy (Statement 2.4)}

Assume that $L(x, u(x), \cdot)$ is strictly quasiconvex at $D u(x)$ a.e. in $\Omega$. We have to show that the convergence in energy property holds, i.e. if $u_{k} \rightarrow u$ in $W^{1,1}$ and $J\left(u_{k}\right) \rightarrow J(u)$ as $k \rightarrow \infty$ then $u_{k} \rightarrow u$ in $W^{1,1}$.

Without loss of generality we can assume that $D u_{k}$ generates a Young measure $\left(v_{x}\right)_{x \in \Omega}$. By Theorem 1.3 we have

$$
\liminf _{k \rightarrow \infty} J\left(u_{k}\right) \geq \int_{\Omega}\left\langle L(x, u(x), \cdot) ; v_{x}\right\rangle d x .
$$

In case $v_{x}$ is nontrivial in a set of positive measure, i.e. in case $D u_{k}$ does not converge strongly in $L^{1}$ (see Lemma 3.10), we have for such points by Lemma 5.2 that

$$
\left\langle L(x, u(x), \cdot) ; v_{x}\right\rangle>L(x, u(x), D u(x)),
$$

when we have the nonstrict inequality a.e. in $\Omega$ by Lemma 5.1 . Then

$$
\liminf _{k \rightarrow \infty} J\left(u_{k}\right)>J(u) .
$$

This contradiction shows that $v_{x}$ should be trivial a.e. in $\Omega$, i.e. by Lemma 3.10 we have $u_{k} \rightarrow u$ in $W^{1,1}$.

Now we have to show that the convergence in energy property implies strict quasiconvexity of $L(x, u(x), \cdot)$ at $D u(x)$ a.e. in $\Omega$.

First we show that $L(x, u(x), \cdot)$ is quasiconvex at $D u(x)$ a.e. in $\Omega$. Assume otherwise. Let $\Omega_{M} \subset \Omega$ be a compact set such that $u: \Omega_{M} \rightarrow \mathbb{R}^{m}$, $D u: \Omega_{M} \rightarrow \mathbb{R}^{m \times n}$ are continuous and $L: \Omega_{M} \times \mathbb{R}^{m} \times \mathbb{R}^{m \times n} \rightarrow \mathbb{R}$ is continuous. If $L(x, u(x), \cdot)$ is not quasiconvex at $D u(x)$ for certain Lebesgue point $x$ of $\Omega_{M}$ we have that for certain homogeneous $L(x, u(x), \cdot)$-gradient Young measure $v$ with compact support there holds

$$
\langle L(x, u(x), \cdot) ; v\rangle<L(x, u(x), D u(x)) .
$$

Define $v_{y}=v \diamond D u(y)$ for $y \in \Omega_{M} \cap B\left(x, \epsilon_{x}\right)$ where $\epsilon_{x}>0$ is so small that

$$
\left\langle L(y, u(y), \cdot) ; v_{y}\right\rangle<L(y, u(y), D u(y)) \text {, for } y \in \Omega_{M} \cap B\left(x, \epsilon_{x}\right) .
$$

Let $z \in \Omega \subset B\left(x, \epsilon_{x}\right)$ be another Lebesgue point of $\Omega_{M}$ and let $v_{1}, v_{2} \in \mathbb{R}^{m \times n}$ be such that $\operatorname{Du}(z) \in\left[v_{1}, v_{2}\right], \operatorname{rank}\left(v_{2}-v_{1}\right)=1$ and $D u(z)=c_{1} v_{1}+c_{2} v_{2}$, where $c_{1}>0, c_{2}>0, c_{1}+c_{2}=1$. Then the measure $v_{z}=c_{1} \delta_{v_{1}}+c_{2} \delta_{v_{2}}$ is a homogeneous $L(z, u(z), \cdot)$-gradient Young measure, see, e.g., [34, Lemma 3.1]. If $v_{1}, v_{2}$ are sufficiently large in modulus we also have

$$
\left\langle L(z, u(z), \cdot) ; v_{z}\right\rangle>L(z, u(z), D u(z))
$$


because of infinite growth of $L$ in $v$. Define $v_{y}=v_{z} \diamond D u(y)$ for $y \in \Omega_{M} \cap B\left(z, \epsilon_{z}\right)$ and take $\epsilon_{x}>0, \epsilon_{z}>0$ to be such that

$$
\int_{\left(B\left(x, \epsilon_{x}\right) \cup B\left(z, \epsilon_{z}\right)\right) \cap \Omega_{M}} L(y, u(y), D u(y)) d y=\int_{\left(B\left(x, \epsilon_{x}\right) \cup B\left(z, \epsilon_{z}\right)\right) \cap \Omega_{M}}\left\langle L(y, u(y), \cdot) ; v_{y}\right\rangle d y .
$$

For $y \in \Omega \backslash\left(\Omega_{M} \cap\left(B\left(x, \epsilon_{x}\right) \cup B\left(z, \epsilon_{z}\right)\right)\right)$ define $v_{y}=\delta_{D u(y)}$. Then the Young measure $\left(v_{y}\right)_{y \in \Omega}$ satisfies all the requirements of Theorem 1.10 and, therefore, is an $L$-gradient Young measure, i.e. there exists a sequence $u_{k} \rightarrow u$ in $W^{1,1}$ such that

$$
\left.J\left(u_{k}\right) \rightarrow \int_{\Omega} L(y, u(y), \cdot) ; v_{y}\right\rangle d y, k \rightarrow \infty .
$$

Due to (5.8) we also have $J\left(u_{k}\right) \rightarrow J(u)$ as $k \rightarrow \infty$, but $u_{k}$ converges only weakly in $W^{1,1}$ by Lemma 3.10. Therefore the convergence in energy property fails at $u$, which shows that $L(x, u(x), \cdot)$ is quasiconvex at $D u(x)$ at all Lebesgue points of $\Omega_{M}$ and, then, a.e. in $\Omega$.

Now we have to show strict quasiconvexity of $L(x, u(x), \cdot)$ at $D u(x)$ for a.e. $x \in \Omega_{M}$. Assume this is not true. Let $\epsilon>0$. Consider a subset $\Omega_{M, \epsilon}$ of $\Omega_{M}$ such that for each $x \in \Omega_{M, \epsilon}$ there exists a homogeneous $L(x, u(x), \cdot)$-gradient Young measure $v$ centered at $D u(x)$ and having the properties:

$$
\begin{gathered}
\langle L(x, u(x), \cdot) ; v\rangle=L(x, u(x), D u(x)), \\
v\left(\mathbb{R}^{m \times n} \backslash B(x, \epsilon)\right) \geq \epsilon,
\end{gathered}
$$

i.e. the strict version of Jensen's inequality fails at $D u(x)$, see Lemma 5.2. Assume that meas $\Omega_{M, \epsilon}>0$ and consider the multi-valued mapping $V: \Omega_{M, \epsilon} \rightarrow\left(M_{1}, \rho\right)$ where $V(x)$ is the set of all homogeneous $L(x, u(x), \cdot)$-gradient Young measures centered at $D u(x)$ and satisfying the requirements $(5.10),(5.11)$. Then the set $\Omega_{M, \epsilon}$ is closed and the mapping $V: \Omega_{M, \epsilon} \rightarrow\left(M_{1}, \rho\right)$ is measurable, even upper semicontinuous, which means that for each sequence $x_{k} \in \Omega_{M, \epsilon}$, for $k \in \mathbb{N}$, with $x_{k} \rightarrow x_{0}$ as $k \rightarrow \infty$ and $v_{k} \in V\left(x_{k}\right)$, for $k \in \mathbb{N}$, with the property $\rho\left(v_{k}, v_{0}\right) \rightarrow 0$ as $k \rightarrow \infty$ we have $\nu_{0} \in V\left(x_{0}\right)$. Indeed we have

$$
\left\langle L\left(x_{0}, u\left(x_{0}\right), \cdot\right) ; v_{0}\right\rangle \leq L\left(x_{0}, u\left(x_{0}\right), D u\left(x_{0}\right)\right)
$$

by validity of (5.10) for $x_{k}$, for $k \in \mathbb{N}$, and by Lemma 3.11. At the same time the property 2) of the integrand $L$ implies that $v_{0}$ is a homogeneous $L\left(x_{0}, u\left(x_{0}\right), \cdot\right)$ gradient Young measure. Therefore Jensen's inequality holds and finally we have the equality

$$
\left\langle L\left(x_{0}, u\left(x_{0}\right), \cdot\right) ; v_{0}\right\rangle=L\left(x_{0}, u\left(x_{0}\right), D u\left(x_{0}\right)\right) .
$$

We obviously also have $v_{0}\left(\mathbb{R}^{m \times n} \backslash B\left(x_{0}, \epsilon\right)\right) \geq \epsilon$, which, together with (5.12) implies $v_{0} \in V\left(x_{0}\right)$. 
Since $V: \Omega_{M, \epsilon} \rightarrow\left(M_{1}, \rho\right)$ is measurable we can select a measurable selection $\tilde{v}: \Omega_{M, \epsilon} \rightarrow\left(M_{1}, \rho\right)$. At other points of $\Omega$ define $\tilde{v}=\delta_{D u(x)}$. By Theorem 1.10 the Young measure $\left(\tilde{v}_{x}\right)_{x \in \Omega}$ is an $L$-gradient Young measure and by (5.11) it is nontrivial. Then for a sequence $D u_{k} \in W^{1,1}\left(\Omega ; \mathbb{R}^{m}\right)$, for $k \in \mathbb{N}$, whose gradient generates $\left(\tilde{v}_{x}\right)_{x \in \Omega}$ as an $L$-gradient Young measure we have that $u_{k}$ converge only weakly to $u$ in $W^{1,1}$ and

$$
J\left(u_{k}\right) \rightarrow \int_{\Omega}\left\langle L(x, u(x), \cdot) ; \tilde{v}_{x}\right\rangle d x=J(u) .
$$

Therefore the convergence in energy property fails for this sequence. This contradiction shows that $L(x, u(x), \cdot)$ is strictly quasiconvex at all points of $\Omega_{M}$ and, then, a.e. in $\Omega$.

\section{3) Relaxation (Statement 2.2)}

First notice that $L^{q c}$ is bounded on compact sets since $L$ is and $L^{q c} \leq L$ everywhere. The integrand $L^{q c}$ has also superlinear growth since for each $\phi \in$ $W_{0}^{1, \infty}\left(\Omega ; \mathbb{R}^{m}\right)$ we have

$$
\int_{\Omega} L(x, u, v+D \phi(y)) d y \geq \int \theta(v+D \phi(y)) d y \geq \theta(v) \text { meas } \Omega,
$$

where $\theta: \mathbb{R}^{m \times n} \rightarrow \mathbb{R}$ is a convex function with superlinear growth such that $L(x, u, v) \geq \theta(v)$ everywhere. Given $x \in \Omega, u \in \mathbb{R}^{m}$ the integrand $L^{q c}(x, u, \cdot)$ is quasiconvex and continuous. This is a standard fact, for a proof see, $e . g .$, [37].

Now we have to show that $L^{q c}$ is a Carathéodory integrand. Let $\tilde{\Omega} \subset \Omega$ be a compact set such that $L: \tilde{\Omega} \times \mathbb{R}^{m} \times \mathbb{R}^{m \times n} \rightarrow \mathbb{R}$ is continuous. We will show that $L^{q c}: \tilde{\Omega} \times \mathbb{R}^{m} \times \mathbb{R}^{m \times n} \rightarrow \mathbb{R}$ is also continuous. First we show lower semicontinuity. Let $x_{k} \rightarrow x_{0}, u_{k} \rightarrow u_{0}, v_{k} \rightarrow v_{0}$ as $k \rightarrow \infty$. By property 2 )

$$
L^{q c}\left(x_{k}, u_{k}, v_{k}\right)=\left\langle L\left(x_{k}, u_{k}, \cdot\right) ; v_{k}\right\rangle
$$

for certain homogeneous $L\left(x_{k}, u_{k}, \cdot\right)$-gradient Young measures $v_{k}$ centered at $v_{k}$, for $k \in \mathbb{N}$. By property 2) there is a subsequence $v_{k}$, for $k \in \mathbb{N}$, (not relabeled) such that $v_{k} \rightarrow^{*} \nu_{0}$, for $k \rightarrow \infty$, and $\nu_{0}$ is a homogeneous $L\left(x_{0}, u_{0}, \cdot\right)$-gradient Young measure centered at $v_{0}$. Then

$$
\liminf _{k \rightarrow \infty} L\left(x_{k}, u_{k}, v_{k}\right)=\liminf _{k \rightarrow \infty}\left\langle L\left(x_{k}, u_{x}, \cdot\right) ; v_{k}\right\rangle \geq\left\langle L\left(x_{0}, u_{0}, \cdot\right) ; v_{0}\right\rangle
$$

by Lemma 3.11. Moreover by Lemma 5.1 we have

$$
\left\langle L\left(x_{0}, u_{0}, \cdot\right) ; v_{0}\right\rangle \geq L^{q c}\left(x_{0}, u_{0}, v_{0}\right) .
$$

The inequalities (5.14), (5.15) imply the inequality

$$
\liminf _{k \rightarrow \infty} L^{q c}\left(x_{k}, u_{k}, v_{k}\right) \geq L^{q c}\left(x_{0}, u_{0}, v_{0}\right),
$$


i.e., lower semicontinuity really holds. Now we show upper semicontinuity. Let $v_{0}$ be a homogeneous $L\left(x_{0}, u_{0}, \cdot\right)$-gradient Young measure centered at $v_{0}$ and such that

$$
L^{q c}\left(x_{0}, u_{0}, v_{0}\right)=\left\langle L\left(x, 0, u_{0}, \cdot\right) ; v_{0}\right\rangle .
$$

Given $\epsilon>0$ there exists a probability measure $\tilde{v}$ of the form $A v\left(v_{0}+D \phi\right)_{\Omega}$, for $\phi \in W^{1, \infty}\left(\Omega ; \mathbb{R}^{m}\right)$, such that

$$
\left|\left\langle L\left(x_{0}, u_{0}, \cdot\right) ; \tilde{v}\right\rangle-\left\langle L\left(x_{0}, u_{0}, \cdot\right) ; v_{0}\right\rangle\right| \leq \epsilon .
$$

Then the measures $v_{k}=\tilde{v} \diamond v_{k}$ are homogeneous $L\left(x_{k}, u_{k}, \cdot\right)$-gradient Young measures centered at $v_{k}$, for $k \in \mathbb{N}$, and, therefore

$$
L^{q c}\left(x_{k}, u_{k}, v_{k}\right) \leq\left\langle L\left(x_{k}, u_{k}, \cdot\right) ; v_{k}\right\rangle \text {, for } k \in \mathbb{N} .
$$

Then (5.16)-(5.18) imply via convergence

$$
\left\langle L\left(x_{k}, u_{k}, \cdot\right) ; v_{k}\right\rangle \rightarrow\left\langle L\left(x_{0}, u_{0}, \cdot\right) ; \tilde{v}\right\rangle, \text { as } k \rightarrow \infty
$$

the inequality

$$
\limsup _{k \rightarrow \infty} L^{q c}\left(x_{k}, u_{k}, v_{k}\right) \leq L^{q c}\left(x_{0}, u_{0}, v_{0}\right)+\epsilon
$$

Therefore upper semicontinuity also holds, i.e. $L^{q c}: \tilde{\Omega} \times \mathbb{R}^{m} \times \mathbb{R}^{m \times n} \rightarrow \mathbb{R}$ is continuous. Then $L^{q c}$ is a Carathéodory integrand.

Let $\tilde{J}$ be the abstract lower semicontinuous envelope of $J$, i.e.

$$
\tilde{J}(u)=\inf \left\{\liminf _{k \rightarrow \infty} J\left(u_{k}\right): u_{k} \rightarrow u \text { in } W^{1,1}\right\}
$$

We have to show that $J^{q c} \leq \tilde{J}$ everywhere and $J^{q c}(u)=\tilde{J}(u)$ for $u \in W^{1,1}\left(\Omega ; \mathbb{R}^{m}\right)$ such that $J(u)<\infty$.

Let $u \in W^{1,1}\left(\Omega ; \mathbb{R}^{m}\right)$ be such that $\tilde{J}(u)<\infty$ and let $u_{k} \in W^{1,1}\left(\Omega ; \mathbb{R}^{m}\right)$, for $k \in \mathbb{N}$, be such that $u_{k} \rightarrow u$ in $W^{1,1}, \lim _{k \rightarrow \infty} J\left(u_{k}\right)=\tilde{J}(u)$. By property 1) we can assume that $D u_{k}$ generates an $L$-gradient Young measure $\left(v_{x}\right)_{x \in \Omega}$. Then by Theorem 1.3 and by Lemma 5.1 we obtain via property 1) that

$$
\begin{aligned}
\lim _{k \rightarrow \infty} J\left(u_{k}\right) & \geq \int_{\Omega}\left\langle L(x, u(x), \cdot) ; v_{x}\right\rangle d x \geq \int_{\Omega} L^{q c}(x, u(x), D u(x)) d x \\
& =J^{q c}(u),
\end{aligned}
$$

i.e. $\tilde{J}(u) \geq J^{q c}(u)$ everywhere.

In case $J(u)<\infty$ we have to show $\tilde{J}(u)=J^{q c}(u)$. Consider the multivalued mapping $V: \Omega \rightarrow\left(M_{1}, \rho\right)$ such that for each $x \in \Omega$ the set $V(x)$ is the set of homogeneous $L(x, u(x), \cdot)$-gradient Young measures centered at $D u(x)$ where 
the infimum of the expression $\langle L(x, u(x), \cdot) ; \nu\rangle$ is attained (this set is nonempty due to property 2)). Then $V$ is a measurable mapping as we have already shown when proving the convergence in energy property. Then by Theorem 3.5 there is a measurable selection $\left(v_{x}\right)_{x \in \Omega}$ of $V$ which is, then, a Young measure by Theorem 3.3. Then by Theorem $1.10\left(v_{x}\right)_{x \in \Omega}$ is an $L$-gradient Young measure, i.e. there exists a sequence $u_{k} \in W^{1,1}\left(\Omega ; \mathbb{R}^{m}\right)$, for $k \in \mathbb{N}$, such that $u_{k} \rightarrow u$ in $W^{1,1}, D u_{k}$ generate $\left(v_{x}\right)_{x \in \Omega}$ as a Young measure and

$$
J\left(u_{k}\right) \rightarrow \int_{\Omega}\left\langle L(x, u(x), \cdot) ; v_{x}\right\rangle d x=J^{q c}(u) .
$$

Therefore $\tilde{J}(u) \leq J^{q c}(u)$, which implies $\tilde{J}(u)=J^{q c}(u)$ since $\tilde{J} \geq J^{q c}$ everywhere.

\section{4) Statement 2.5}

Let $f \in W^{1,1}\left(\Omega ; \mathbb{R}^{m}\right)$ be such that $J(f)<\infty$. Consider the set

$$
S=\left\{u \in W^{1,1}\left(\Omega ; \mathbb{R}^{m}\right):\left.u\right|_{\partial \Omega}=\left.f\right|_{\partial \Omega}\right\} .
$$

This set satisfies all of the assumptions of Theorem 1.9. Then there exists a set $V \subset S^{\prime}$, where $S^{\prime}=\{u \in S: J(u)<\infty\}$, such that the functional $J: S^{\prime} \rightarrow \mathbb{R}$ is lower semicontinuous and admits the convergence in energy property at elements of the set $V$. Then the functional $\tilde{J}: \bar{S}^{\prime} \rightarrow \overline{\mathbb{R}}$ (here $\bar{S}^{\prime}$ is the closure of $S^{\prime}$ in the weak topology of $W^{1,1}$ ), which is the lower semicontinuous envelope of the functional $J: S^{\prime} \rightarrow \mathbb{R}$ with respect to the weak topology in $W^{1,1}$ also admits the convergence in energy property at the elements of $V$, see Theorem 1.9. Moreover given $u \in \bar{S}^{\prime}$ with $\tilde{J}(u)<\infty$ there exists a sequence $u_{k} \in V$ such that $u_{k} \rightarrow u$ in $W^{1,1}$ and $J\left(u_{k}\right) \rightarrow \tilde{J}(u)$, as $k \rightarrow \infty$. Therefore to prove Theorem 2.5 we need only show that for $u \in V$ the functions $L(x, u(x), \cdot), L^{q c}(x, u(x), \cdot)$ are strictly quasiconvex at $D u(x)$ for a.e. $x \in \Omega$. In the case of functional $J$ this property really holds since Theorem 2.4 is valid. A subtler issue is to prove this result in the case of $J^{q c}$.

Consider a compact subset $\tilde{\Omega}$ of $\Omega$ such that $u: \tilde{\Omega} \rightarrow \mathbb{R}^{m}, D u: \tilde{\Omega} \rightarrow \mathbb{R}^{m \times n}$ are continuous and $L: \tilde{\Omega} \times \mathbb{R}^{m} \times \mathbb{R}^{m \times n} \rightarrow \mathbb{R}$ is also continuous. Assume that this is not true, i.e., that $L^{q c}(x, u(x), \cdot)$ is strictly quasiconvex at $D u(x)$ for a.e. $x \in \tilde{\Omega}$. Then for certain $\epsilon>0$ the subset $\Omega_{\epsilon}$ of $\tilde{\Omega}$ consisting of points $x \in \tilde{\Omega}$ such that there exists a sequence $\phi_{k} \in W_{0}^{1, \infty}\left(\Omega ; \mathbb{R}^{m}\right)$ with the properties

$$
\begin{gathered}
\text { meas }\left\{y \in \Omega:\left|D \phi_{k}(y)\right| \geq \epsilon\right\} \geq \epsilon, \text { for } k \in \mathbb{N}, \\
\int_{\Omega} L^{q c}\left(x, u(x), D u(x)+D \phi_{k}(y)\right) d y \rightarrow L(x, u(x), D u(x)) \text { meas } \Omega, \text { as } k \rightarrow \infty,(
\end{gathered}
$$

has positive measure. 
Given $j \in \mathbb{N}$ and a Lebesgue point $x_{0} \in \Omega_{\epsilon}$ there exists a function $\phi \in$ $W_{0}^{1, \infty}\left(\Omega ; \mathbb{R}^{m}\right)$ such that

$$
\begin{gathered}
\text { meas }\{y \in \Omega:|D \phi(y)| \geq \epsilon\} \geq \epsilon, \\
\mid \int_{\Omega} L(x, u(x), D u(x) \quad+D \phi(y)) d y-L(x, u(x), D u(x)) \text { meas } \Omega \mid<1 / j .
\end{gathered}
$$

Define $v_{x_{0}}^{j}=A v\left(D u\left(x_{0}\right)+D \phi\right)_{\Omega}$ and define $v_{x}^{j}=D u(x) \diamond v_{x_{0}}^{j}$ for $x \in \Omega_{\epsilon} \cup$ $B\left(x_{0}, \delta\right)$ where $\delta>0$ is so small that the inequality (5.22) holds also for $x, v_{x}^{j}$ (this is true due to the continuity assumptions and due to the compactness of the support of $\left.v_{x_{0}}\right)$. Then the set $\Omega_{\epsilon}$ can be decomposed into subsets $B\left(x_{i}, \epsilon_{i}\right) \cap \Omega_{\epsilon}$, for $i \in \mathbb{N}$, where (5.22) holds and a set of zero measure. Then a Young measure $\left(v_{x}^{j}\right)_{x \in \Omega}$ is defined in the set $\cup_{i=1}^{\infty} B\left(x_{i}, \epsilon_{i}\right) \cap \Omega_{\epsilon}$ and assume that $v_{x}^{j}=\delta_{D u(x)}$ everywhere else. By Theorem $1.10\left(v_{x}^{j}\right)_{x \in \Omega}$ is an $L^{q c}$-gradient Young measure. To see this we need only verify property (h4) among the conditions of Theorem 1.10. There exists an almost piece-wise affine sequence $u_{k} \rightarrow u$ in $W^{1,1}$ such that $J\left(u_{k}\right) \rightarrow J(u)$, as $k \rightarrow \infty$. By Theorem 1.3 the sequence $L\left(\cdot, u_{k}(\cdot), D u_{k}(\cdot)\right)$, for $k \in \mathbb{N}$, is equi-integrable. Since $L^{q c} \leq L$ everywhere the sequence $L^{q c}\left(\cdot, u_{k}(\cdot), D u_{k}(\cdot)\right)$, for $k \in \mathbb{N}$, is also equi-integrable and, then, $J^{q c}\left(u_{k}\right) \rightarrow J^{q c}(u)$, as $k \rightarrow \infty$ by the same theorem. Therefore the conclusion of Theorem 1.10 holds for $J^{q c}$ and, therefore, $\left(v_{x}^{j}\right)_{x \in \Omega}$ is an $L^{q c}$-gradient Young measure. We also have that a subsequence $\left(v_{x}^{j}\right)_{x \in \Omega}$, for $j \in \mathbb{N}$, (not relabeled) generates a Young measure $\left(\tilde{v}_{x}\right)_{x \in \Omega}$ which is not trivial. On the other hand

$$
\int_{\Omega}\left\langle L(x, u(x), \cdot) ; v_{x}^{j}\right\rangle d x \rightarrow J^{q c}(u), j \rightarrow \infty .
$$

If $u_{l}^{j}, l \in \mathbb{N}$, are sequences whose gradients generate $\left(v_{x}^{j}\right)_{x \in \Omega}$, for $j \in \mathbb{N}$, as $L^{q c}$ gradient Young measures then for an appropriate subsequence $u_{l(j)}^{j} \in W^{1,1}\left(\Omega ; \mathbb{R}^{m}\right)$, for $j \in \mathbb{N}$, we have that $D u_{l(j)}^{j}$, for $j \in \mathbb{N}$, generates $\left(\tilde{v}_{x}\right)_{x \in \Omega}$ as a Young measure and by (5.23) we also have

$$
J^{q c}\left(u_{l(j)}^{j}\right) \rightarrow J^{q c}(u), \text { as } j \rightarrow \infty .
$$

Therefore the convergence in energy property fails for the functional $J^{q c}$ at the function $u$, which is a contradiction. This way we showed that $L^{q c}(x, u(x), \cdot)$ is strictly quasiconvex at $D u(x)$ a.e. in $\Omega$. Therefore Statement 2.5 is valid.

Now all the results of Theorem 2.6 are proved.

To complete the section we want to mention that in the scalar case $(m=1)$ the minimization problems that admits the attainment property can be completely characterized.

The following theorem was first proved by Cellina, see $[9,10]$, and independently by Friesecke, see [23]. 
Theorem 5.3. Let $L: \mathbb{R}^{n} \rightarrow \overline{\mathbb{R}}$ be a continuous integrand and let $A \in \mathbb{R}^{n}$. Then the minimization problem

$$
J(u) \rightarrow \min , u \in W^{1,1}(\Omega),\left.u\right|_{\partial \Omega}=\left.l_{A}\right|_{\partial \Omega},
$$

admits a solution if and only if either $\partial L(A) \neq \varnothing$ or there exist $v_{1}, \ldots, v_{q}$ such that $A$ belongs to the interior of the convex hull of the set $\left\{v_{1}, \ldots, v_{q}\right\}$ and $\cap_{i=1}^{q} \partial L\left(v_{i}\right) \neq \emptyset$.

If the condition on $L$ described in Theorem 5.3 holds everywhere then the attainment result holds for arbitrary boundary-valued minimization problem provided the convexified problem admits at least one solution which is differentiable in the classical sense a.e. in $\Omega$, i.e., the following theorem is valid.

Theorem 5.4 ([47]). Let $L: \mathbb{R}^{n} \rightarrow \overline{\mathbb{R}}$ be a continuous integrand with superlinear growth. Assume that the condition on $L$ from Theorem 5.3 holds for each $A \in \mathbb{R}^{n}$ and assume $f \in W^{1,1}$ is such that as above $J(f)<\infty$. Then the problem

$$
J(u) \rightarrow \min ,\left.u\right|_{\partial \Omega}=\left.f\right|_{\partial \Omega}, u \in W^{1,1}(\Omega),
$$

admits a minimizer provided the minimization problem

$$
J^{c}(u)=\int_{\Omega} L^{* *}(D u(x)) d x \rightarrow \min ,\left.u\right|_{\partial \Omega}=\left.f\right|_{\partial \Omega}, u \in W^{1,1}(\Omega),
$$

admits a solution which is differentiable in the classical sense a.e. in $\Omega$ (here $L^{* *}$ is the convexification of $L$ ).

In the case of strong materials, i.e., when $L \geq \alpha|\cdot|^{n+\epsilon}+\beta, \alpha>0, \epsilon>0$, all admissible functions for problems (5.24), (5.25) belong to the space $W^{1, n+\epsilon}(\Omega)$ and, therefore, are differentiable in the classical sense a.e. in $\Omega$, see [18]. Celada and Perrotta, see [12], showed following a suggestion by Sverak a.e. differentiability of minimizers in the case case of problems with $p$-growth. One more case when we can claim a.e. differentiability in the classical sense of at least one solution of (5.25) is discussed in [40]. In order to complete the results on attainment in the scalar case we have to show validity of the following conjecture

Conjecture 3. Let $L: \mathbb{R}^{n} \rightarrow \overline{\mathbb{R}}$ be a convex integrand with superlinear growth and assume $f \in W^{1,1}(\Omega)$ to be such that $J(f)<\infty$. Then for the minimization problem

$$
J(u) \rightarrow \min ,\left.u\right|_{\partial \Omega}=\left.f\right|_{\partial \Omega}, u \in W^{1,1}(\Omega),
$$

there exists at least one solution which is diferentiable in the classical sense a.e. in $\Omega$. 


\section{Proof of Theorems 2.7 and 2.8}

Problems with $p(x)$-growth attracted wide attention of specialists in the last decade, see, e.g., [31]. The weak convergence theory for such problems was studied in [1, $13,32,33,42,43$ ] for the case when $p(\cdot)$ is continuous and the modulus of continuity satisfies so-called Zhikov's condition, see [52]. As we see Theorem 2.7 asserts that the whole theory is valid for arbitrary measurable functions $p(\cdot)$ in the case of strong materials that shows another advantage of strong materials.

In the proof of Theorems $2.7,2.8$ we will use the following crucial result due to Kristensen, [26]. This result was first published in [27].

Theorem 6.1. Let $v$ be a homogeneous 1-gradient Young measure such that $\langle$. $\left|{ }^{p} ; v\right\rangle\langle\infty$, with $p>1$. Then $v$ is a homogeneous p-gradient Young measure.

Proof of Theorem 2.7. It is easy to see that the properties 1), 2) of Section 2 are valid because of the Kristensen's Theorem 6.1. Therefore we need only show the validity of the approximation property 3$)$.

Let $x_{0} \in \Omega$ be a Lebesgue point of $D u(\cdot)$ and a point of classical differentiability of $u(\cdot)$. Let $i \in\{1, \ldots, m\}$ and consider the $i$-th component $u^{i}(\cdot)$ of the function $u: \Omega \rightarrow \mathbb{R}^{m}$. Given $\epsilon>0, s>0$ consider a function

$$
\tilde{u}^{i}(x)=u^{i}\left(x_{0}\right)+\left\langle D u^{i}\left(x_{0}\right), x-x_{0}\right\rangle+\phi_{\epsilon, s}\left(x-x_{0}\right),
$$

where

$$
\phi_{\epsilon, s}\left(x-x_{0}\right)=s-\max _{v \in S\left(D u^{i}\left(x_{0}\right), \epsilon\right)}\left\langle v, x-x_{0}\right\rangle,
$$

where $S\left(D u^{i}\left(x_{0}\right), \epsilon\right)$ is a sphere centered at $D u^{i}\left(x_{0}\right)$ and with radius $\epsilon$. Note that $\phi_{\epsilon, s}(\cdot)>0$ in the set $B(x, s / \epsilon)$ and $\phi_{\epsilon, s}(\cdot)=0$ in $S\left(D u^{i}\left(x_{0}\right), s / \epsilon\right)$. We also have $\left|\tilde{u}^{i}(x)-\tilde{u}^{i}\left(x_{0}\right)\right|=-\epsilon\left|x-x_{0}\right|$ and, therefore, $\tilde{u}^{i} \geq u^{i}$ in the set $\Omega_{s, \epsilon}^{i}\left(x_{0}\right)$ such that

$$
B\left(x_{0}, s / \epsilon-w^{i}(s / \epsilon)\right) \subset \Omega_{s, \epsilon}^{i}\left(x_{0}\right) \subset B\left(x_{0}, s / \epsilon+w^{i}(s / \epsilon)\right),
$$

where $w^{i}(t) \rightarrow 0$ as $t \rightarrow 0$ due to classical differebtiability of $u^{i}$ at $x_{0}$.

Let $w(\cdot)=\max _{1 \leq i \leq m} w_{i}(\cdot)$. Then

$$
D \tilde{u}(x)=D u\left(x_{0}\right)+D \phi(x), \text { for } x \in \Omega_{s, \epsilon}\left(x_{0}\right)=\cap_{i=1}^{m} \Omega_{s, \epsilon}^{i}\left(x_{0}\right),
$$

where

$$
B\left(x_{0}, s / \epsilon-w(s / \epsilon)\right) \subset \Omega_{s, \epsilon}\left(x_{0}\right) \subset B\left(x_{0}, s / \epsilon+w(s / \epsilon)\right) .
$$

In the set $\Omega_{s, \epsilon}\left(x_{0}\right)$ we have the following simple estimate

$$
|D \tilde{u}(x)| \leq\left|D u\left(x_{0}\right)\right|+\epsilon .
$$

Now we obtain the estimate on $D \tilde{u}$ in the set $\cup_{i=1}^{m} \Omega_{s, \epsilon}^{i}\left(x_{0}\right) \backslash \Omega_{s, \epsilon}\left(x_{0}\right)$. In case $|D \tilde{u}(x)| \leq 2|D u(x)|$ we have

$$
|D \tilde{u}(x)|^{p(x)} \leq 2^{p(x)}|D u(x)|^{p(x)} \leq 2^{p_{2}}|D u(x)|^{p(x)} .
$$


In case $|D \tilde{u}(x)| \geq 2|D u(x)|$ we have $|v(x)|+|D u(x)| \geq 2|D u(x)|$ and then $2|v(x)| \geq|D \tilde{u}(x)|$, i.e.

$$
|D \tilde{u}(x)|^{p(x)} \leq 2^{p(x)}|v(x)|^{p(x)} \leq 2^{p_{2}}\left(\left|D u\left(x_{0}\right)\right|+\epsilon\right)^{p(x)} .
$$

Now we construct a sequence $u_{k} \in u+W_{0}^{1,1}\left(\Omega ; \mathbb{R}^{m}\right)$ with the property 3$)$. For $k \in \mathbb{N}$ consider a finite collection of disjoint balls $B\left(x_{j}, s_{j} / \epsilon_{j}+w_{j}\left(s_{j} / \epsilon_{j}\right)\right)$, $j \in\{1, \ldots, j(k)\}$, such that (6.3) holds with $w_{j}\left(s_{j} / \epsilon_{j}\right) \leq 1 / k$ and $\epsilon_{j} \leq 1 / k$, $j \in\{1, \ldots, j(k)\}$, and meas $\left(\Omega \backslash \cup_{j=1}^{j(k)} \Omega_{s_{j}, \epsilon_{j}}\left(x_{j}\right)\right) \leq 1 / k$. Then $s_{j}, \epsilon_{j}, j \in$ $\{1, \ldots, j(k)\}$, can be also selected in such a way that

$$
\begin{aligned}
& \left|\int_{\Omega_{s_{j}, \epsilon_{j}\left(x_{j}\right)}} L(y, u(y), D u(y)) d y-\int_{\Omega_{s_{j}, \epsilon_{j}}\left(x_{j}\right)} L(y, \tilde{u}(y), D \tilde{u}(y)) d y\right| \\
& \quad \leq 1 / k \text { meas } \Omega_{s_{j}, \epsilon_{j}}\left(x_{j}\right), j \in\{1, \ldots, j(k)\},
\end{aligned}
$$

see (6.4), and, due to (6.5), (6.6), we also have

$$
\begin{aligned}
& \int_{B\left(x_{j}, s_{j} / \epsilon_{j}+w_{j}\left(s_{j} / \epsilon_{j}\right)\right) \backslash \Omega_{s_{j}, \epsilon_{j}}\left(x_{j}\right)}|L(y, \tilde{u}(y), D \tilde{u}(y))| d y \\
& \leq c \int_{B\left(x_{j}, s_{j} / \epsilon_{j}+w_{j}\left(s_{j} / \epsilon_{j}\right)\right) \backslash \Omega_{s_{j}, \epsilon_{j}}\left(x_{j}\right)} 2^{p_{2}+1}\{\mid L(u, u(y), D u(y) \mid+1\} d y .
\end{aligned}
$$

Therefore the function $u_{k}$ such that $u_{k}^{i}=\tilde{u}^{i}$ in $\Omega_{s_{j}, \epsilon_{j}}^{i}\left(x_{j}\right)$, for $i \in\{1, \ldots, m\}$, $j \in\{1, \ldots, j(k)\}$, satisfies the properties

$$
\begin{gathered}
u_{k}=\tilde{u}, x \in \Omega_{s_{j}, \epsilon_{j}}\left(x_{j}\right), \text { for } j \in\{1, \ldots, j(k)\}, \\
\int_{\Omega}\left|L\left(y, u_{k}(y), D u_{k}(y)\right)-L(y, u(y), D u(y))\right| d y \leq 1 / k \text { meas } \Omega \\
+c \sum_{j=1}^{j(k)} 2^{p_{2}+1} \int_{\cup_{j}\left(B\left(x_{j}, s_{j} / \epsilon_{j}+w_{j}\left(s_{j} / \epsilon_{j}\right)\right) \backslash B\left(x_{j}, s_{j} / \epsilon_{j}-w_{j}\left(s_{j} / \epsilon_{j}\right)\right)\right)} \\
\{|L(y, u(y), D u(y))|+1\} d y,
\end{gathered}
$$

see (6.7) and (6.8). Then $s_{j}, \epsilon_{j}$ can be selected in such a way that due to (6.3), (6.4), (6.7), (6.8) (see also (6.9) and (6.10)) we obtain

$$
\begin{aligned}
& \left\|u_{k}-u\right\|_{W^{1,1}\left(\Omega ; \mathbb{R}^{m}\right)} \rightarrow 0 \\
& \left\|L\left(\cdot, u_{k}(\cdot), D u_{k}(\cdot)\right)-L(\cdot, u(\cdot), D u(\cdot))\right\|_{L^{1}(\Omega)} \rightarrow 0, \text { as } k \rightarrow \infty
\end{aligned}
$$

and

$$
\operatorname{meas}\left(\Omega \backslash \cup_{j=1}^{j(k)} \Omega_{s_{j}, \epsilon_{j}}\left(x_{j}\right)\right) \leq 1 / k \text { meas } \Omega \text {. }
$$


Finally we can approximate $u_{k}$ by piece-wise affine functions $\tilde{u}_{k} \in u_{k}+W_{0}^{1, \infty} \times$ $\left(\cup_{j=1}^{j(k)} \Omega_{s_{j}, \epsilon_{j}}\left(x_{j}\right) ; \mathbb{R}^{m}\right)$ such that due to (6.11) and (6.12) we have

$$
\begin{aligned}
& \left\|\tilde{u}_{k}-u\right\|_{W^{1,1}} \rightarrow 0 \\
& \left\|L\left(\cdot, \tilde{u}_{k}(\cdot), D \tilde{u}_{k}(\cdot)\right)-L(\cdot, u(\cdot), D u(\cdot))\right\|_{L^{1}} \rightarrow 0, k \rightarrow \infty .
\end{aligned}
$$

The sequence $\tilde{u}_{k} \in W^{1, n+\epsilon}\left(\Omega ; \mathbb{R}^{m}\right)$ is an almost piece-wise affine sequence with the properties

$$
\left\|\tilde{u}_{k}-u\right\|_{W^{1,1}} \rightarrow 0, \quad J\left(\tilde{u}_{k}\right) \rightarrow J(u), \text { for } k \rightarrow \infty
$$

This proves validity of the property 3 ) of Section 2 . Therefore Theorem 2.7 follows from Theorem 2.6.

Proof of Theorem 2.8. relies on the same arguments as the proof of Theorem 2.7.

Note that the weak convergence theory is established for integral functionals with integrands $L$ satisfying the estimates

$$
c_{1} G(x, u, v)+c_{2} \leq L(x, u, v) \leq c_{3} G(x, u, v)+c_{4}, \quad c_{3} \geq c_{1}>0,
$$

where for fixed $(x, u)$ the function $G(x, u, \cdot)$ has $p$-growth, see (1.2). Of course one wants to extend the theory to a larger class of integrands, say for $G(x, u, v)$ convex in $v$ and with superlinear growth in $v$. To do this we need a positive answer to the following

Conjecture 4. Let $L: \mathbb{R}^{m \times n} \rightarrow \mathbb{R}$ be a convex integrand with superlinear growth. Let also $L_{k}$, for $k \in \mathbb{N}$, be an increasing sequence of convex integrands with superlinear growth such that $L_{k} \rightarrow L$, as $k \rightarrow \infty$, uniformly on compact sets. Then given $\Phi \in C_{c}^{\infty}\left(\mathbb{R}^{m \times n}\right)$ and $A \in \mathbb{R}^{m \times n}$ we have

$$
\left(L_{k}+\Phi\right)^{q c}(A) \rightarrow(L+\Phi)^{q c}(A), \text { as } k \rightarrow \infty .
$$

Validity of Conjecture 4 would allow to extend Kristensen's result Theorem 6.1 to the case of arbitrary convex integrands $L$, i.e., we would prove that every homogeneous 1-gradient Young measure $v$ with $\langle L ; v\rangle<\infty$ is a homogeneous $L$ gradient Young measure. Indeed the sequence $L_{k}$ can be selected in such a way that $v$ is a homogeneous $L_{k}$-gradient Young measure, for $k \in \mathbb{N}$, and, in particular, $\left\langle L_{k} ; v\right\rangle\left\langle\infty\right.$, for $k \in \mathbb{N}$. Then $\left\langle L_{k}+\Phi ; v\right\rangle \geq\left(L_{k}+\Phi\right)^{q c}(A)$, for $k \in \mathbb{N}$. Since $\left(L_{k}+\Phi\right)^{q c}(A) \rightarrow(L+\Phi)^{q c}(A)$ and $\left\langle L_{k}+\Phi ; v\right\rangle \rightarrow\langle L+\Phi ; v\rangle$ as $k \rightarrow \infty$ we obtain that $\langle L+\Phi ; v\rangle \geq(L+\Phi)^{q c}(A)$ and, then, by Theorem $1.8 v$ is a homogeneous $L$-gradient Young measure. Therefore properties 1), 2) from Section 2 hold and, then, lower semicontinuity and the convergence in energy property are valid. To prove Theorems 2.2, 2.5 we need also property 3 ) which is a separate issue. 
Therefore validity of Conjecture 4 would allow to extend the weak convergence theory to a much larger class of integrands. At the moment it is known that this conjecture is valid in the case of $L$ with $p$-growth. We explain this. For $k \in \mathbb{N}$, $A \in \mathbb{R}^{m \times n}$ there is a sequence of homogeneous $\left(L_{k}+\Phi\right)$-gradient Young measures $v_{k}^{j}$, for $j \in \mathbb{N}$, centered at $A$ and such that

$$
\left\langle L_{k}+\Phi ; v_{k}^{j}\right\rangle \rightarrow\left(L_{k}+\Phi\right)^{q c}(A), \text { as } j \rightarrow \infty,
$$

see Lemma 5.1. Without loss of generality we can assume that $v_{k}^{j} \rightarrow^{*} v_{k}$, as $j \rightarrow \infty$. Then $v_{k}$ is a homogeneous 1 -gradient Young measure and

$$
\liminf _{j \rightarrow \infty}\left\langle L_{k}+\Phi ; v_{k}^{j}\right\rangle \geq\left\langle L_{k}+\Phi ; v_{k}\right\rangle
$$

due to Lemma 3.11. However we do not know whether $v_{k}$ is a homogeneous $\left(L_{k}+\right.$ $\Phi)$-gradient Young measure since the validity of property 2) from Section 2 is not known. In any case for a subsequence (not relabeled) such that $v_{k} \rightarrow^{*} v$, as $k \rightarrow \infty$, and $\langle L ; v\rangle<\infty$ we have by Theorem 6.1 that $v$ is a homogeneous $L$-gradient Young measure. Then

$$
\left\langle L_{k}+\Phi ; v_{k}\right\rangle \leq\left(L_{k}+\Phi\right)^{q c}(A) \leq(L+\Phi)^{q c}(A) \leq\langle L+\Phi ; v\rangle
$$

and since by Lemma 3.11

$$
\liminf _{k \rightarrow \infty}\left\langle L_{k}+\Phi ; v_{k}\right\rangle \geq\langle L+\Phi ; v\rangle, \text { as } k \rightarrow \infty,
$$

we infer that

$$
\left(L_{k}+\Phi\right)^{q c}(A) \rightarrow(L+\Phi)^{q c}(A), \text { as } k \rightarrow \infty .
$$

Therefore Conjecture 4 is valid. Note also that $L_{k}$, for $k \in \mathbb{N}$, could be only continuous integrands, i.e. convexity is not required for the validity of the result.

We have to notice that Conjecture 4 fails if $L$ is only quasiconvex and $L_{k}$, for $k \in \mathbb{N}$, are only continuous. To see this we can use the example due to Ball and Murat discussed in Section 2. In this example $L=\mu|D u|^{n-\epsilon}+|\operatorname{Det} D u|$, $\Omega=B(0,1)$ and for $L$-gradient Young measure $v=A v(D(x /|x|))_{\Omega}$ centered at Id we have $\langle L ; v\rangle<L$ (Id). Therefore we can not claim that $\left(L_{k}+\Phi\right)^{q c}$ (Id) $\rightarrow$ $(L+\Phi)^{q c}(\mathrm{Id})$ for $\Phi=0$ since $L_{k}^{q c}(\mathrm{Id}) \leq\left\langle L_{k} ; \nu\right\rangle \leq\langle L ; \nu\rangle<L$ (Id) for all $k \in \mathbb{N}$. In fact there are simply no sequences of quasiconvex integrands $L_{k}$ with $(n-\epsilon)$-growth which converge to $L$ uniformly on compact sets.

However in the case of strong materials we still can hope to prove the result which is:

Conjecture 5. Let $L: \mathbb{R}^{m \times n} \rightarrow \mathbb{R}$ be quasiconvex and such that $L(v) \geq \alpha|v|^{n+\epsilon}+$ $\beta$, where $\alpha>0, \epsilon>0$. Let also $L_{k}: \mathbb{R}^{m \times n} \rightarrow \mathbb{R}$ be an increasing sequence of integrands with superlinear growth and such that $L_{k} \rightarrow L$, as $k \rightarrow \infty$, uniformly on compact sets. Then for each $A \in \mathbb{R}^{m \times n}$ and each $\Phi \in C_{c}^{\infty}\left(\mathbb{R}^{m \times n}\right)$ we have

$$
\left(L_{k}+\Phi\right)^{q c}(A) \rightarrow(L+\Phi)^{q c}(A), \text { as } k \rightarrow \infty .
$$


The validity of Conjecture 5 would imply the validity of both the Conjectures 1) and 2).

Note that although the situation with strong materials was explicitely discussed only in our recent paper [37] it already attracted attention of some specialists and in [2] the authors studied the case of strong materials in context of the homogenization theory.

Proving Conjectures 4 and 5 would greatly extend the weak convergence theory and we hope to address this issue in subsequent papers.

\section{References}

[1] E. Acerbi, G. Bouchitté and I. Fonseca, Relaxation of convex functionals: the gap problem, Ann. Inst. H. Poincaré, Anal. Non Linéaire 20 (2003), 359-390.

[2] O. AnZA Hafsa and J.-P. Mandallena, Homogenization of nonconvex integrals with convex growth, J. Math. Pures Appl. (9) 96 (2011), 167-189.

[3] J. M. BALL, Convexity conditions and existence theorems in nonlinear elasticity, Arch. Ration. Mech. Anal. 63 (1976/77), 337-403.

[4] J. M. BALL, A version of the fundamental theorem for Young measures, In: "PDEs and Continuum Models of Phase Transitions" (Nice, 1988), Lecture Notes in Phys., Vol. 344, Springer, Berlin, 1989, 207-215.

[5] E. J. BALDER, A general approach to lower semicontinuity and lower closure in optimal control theory, SIAM J. Control Optim. 22 (1984), 570-598.

[6] J. M. BALL and F. MURAT, $W^{1, p}$-quasiconvexity and variational problems for multiple integrals, J. Funct. Anal. 58 (1984), 225-253.

[7] N. N. Bogolubov, Sue quelques methodes nouvelles dans calcul des variations, Ann. Mat. Pura Appl. 7 (1930), 149-271.

[8] A. Braides and A. Defranceschi, 'Homogenization of Multiple Integrals", Oxford Lecture Series in Mathematics and its Applications, 12, The Clarendon Press, Oxford University Press, New York, 1998. xiv+298 pp.

[9] A. Cellina, On minima of a functional of the gradient: necessary conditions, Nonlinear Anal. 20 (1993), 337-341.

[10] A. CELlina, On minima of a functional of the gradient: sufficient conditions, Nonlinear Anal. 20 (1993), 343-347.

[11] C. Castaing and M. Valadier, "Convex Analysis and Measurable Multifunctions", Lecture Notes in Mathematics, Vol. 580. Springer-Verlag, Berlin-New York, 1977.

[12] P. CELADA and S. PERrotTA, Minimizing non-convex multiple integrals: a density result, Proc. Roy. Soc. Edinburgh Sect. A 130 (2000), 721-741.

[13] A. CosCIA and D. MUCCI, Integral representation and $\Gamma$-convergence of variational integrals with $P(X)$-growth, ESAIM Control Optim. Calc. Var. 7 (2002), 495-519.

[14] B. DACOROGNA, Quasiconvexity and relaxation of nonconvex problems in the calculus of variations, J. Funct. Anal. 46 (1982), 102-118.

[15] E. De Giorgi. Sulla convergenza di alcune successioni d'integrali del tipo dell'area, Collection of articles dedicated to Mauro Picone on the occasion of his nintieth birthday, Rend. Mat. 8 (1975), $277-294$.

[16] L. C. EVANS and R. F. GARIEPY, Some remarks concerning quasiconvexity and strong convergence, Proc. Roy. Soc. Edinburgh Sect. A 106 (1987), 53-61.

[17] L. C. EVANS and R. F. GARIEPY, Blowup, compactness and partial regularity in the calculus of variations, Indiana Univ. Math. J. 36 (1987), 361-371.

[18] L. C. Evans and R. F. GARIEPY, "Measure Theory and Fine Properties of Functions", Studies in Advanced Mathematics, CRC Press, Boca Raton, FL, 1992. 
[19] A. A. Egorov, Quasiconvex functions and null Lagrangians in problems of the stability of classes of mappings (Russian) Sibirsk. Mat. Zh. 49 (2008), 796-812; translation in Sib. Math. J. 49 (2008), 637-649.

[20] I. FonsECA and J. MALY, Ralaxation of multiple integrals below the growth exponent, Ann. Inst. H. Poincaré, Anal. Non Linéaire 14 (1997), 309-338.

[21] I. Fonseca, J. Maly and G. Mingione, Scalar minimizers with fractal singular sets, Arch. Ration. Mech. Anal. 172 (2004), 295-307.

[22] M. Foss, W. J. HrusA and V. J. Mizel, The Lavrentiev gap phenomenon in nonlinear elasticity, Arch. Ration. Mech. Anal. 167 (2003), 337-365.

[23] G. FRIESECKE, A necessary and sufficient condition for nonattainment and formation of microstructure almost everywhere in scalar variational problems, Proc. Roy. Soc. Edinburgh Sect. A 124 (1994), 437-471.

[24] D. Kinderlehrer and P. Pedregal, Gradient Young measures generated by sequences in Sobolev spaces, J. Geom. Anal. 4 (1994), 59-90.

[25] D. Kinderlehrer and P. Pedregal, Weak convergence of integrands and the Young measure representation, SIAM J. Math. Anal. 23 (1992), 1-19.

[26] J. KRISTEnsen, "Finite Functionals and Young Measures Generated by Gradients of Sobolev Functions", Thes. Doct. Phylosophy, Math. Inst., Technical University of Denmark, Lyngby, Denmark 1994.

[27] J. KRISTENSEN, Lower semicontinuity in spaces of weakly differentiable functions, Math. Ann. 313 (1999), 653-710.

[28] K. KuRATOWSKI and C. RYLL-NARDZEWSKI, A general theorem on selectors, Bull. Acad. Polon. Sci. 13 (1966), 397-403.

[29] C. B. Morrey, Quasi-convexity and the lower semicontinuity of multiple integrals, Pacific J. Math. 2 (1952), 25-53.

[30] J.-P. Mandallena, Lower semicontinuity via $W^{1, p}$-quasiconvexity, Bull. Sci. Math. 137 (2013), 602-616.

[31] G. MingIOnE, Regularity of minima: an invitation to the dark side of the calculus of variations, Appl. Math. 51 (2006), 355-426.

[32] G. MingIONE and D. MUCCI, Integral functionals and the gap problem: sharp bounds for relaxation and energy concentration, SIAM J. Math. Anal. 36 (2005), 1540-1579 (electronic).

[33] D. MuccI, Relaxation of variational functionals with piecewise constant growth conditions, J. Convex Anal. 10 (2003), 295-324.

[34] S. MulLER and M. A. SYCHEV, Optimal existence theorems for nonhomogeneous differential inclusions, J. Funct. Anal. 181 (2001), 447-475.

[35] M. A. SYCHEV, Young measures as measurable functions and their applications to variational problems (Russian) Zap. Nauchn. Sem. S.-Peterburg. Otdel. Mat. Inst. Steklov. (POMI) 310 (2004), Kraev. Zadachi Mat. Fiz. i Smezh. Vopr. Teor. Funkts. 35 [34], 191212, 228-229; translation in J. Math. Sci. (N. Y.) 132 (2006), 359-370.

[36] M. A. SYCHEV, A new approach to Young measure theory, relaxation and convergence in energy, Ann. Inst. H. Poincaré Anal. Non Linéaire 16 (1999), 773-812.

[37] M. A. SYCHEV, First general lower semicontinuity and relaxation results for strong materials, J. Convex Anal. 17 (2010), 183-202.

[38] M. A. Sychev, Characterization of homogeneous gradient Young measures in case of arbitrary integrands, Ann. Sc. Norm. Sup. Pisa Cl. Sci. (4) 29 (2000), 531-548.

[39] M. A. SYCHEV, Young measure approach to characterization of behaviour of integral functionals on weakly convergent sequences by means of their integrands, Ann. Inst. H. Poincaré, Anal. Non Linéaire 15 (1998), 755-782.

[40] M. A. SYCHEV, Attainment and relaxation results in special classes of deformations, Calc. Var. Partial Differential Equations 19 (2004), 183-210. 
[41] M. A. SYCHEV, Semicontinuity and relaxation theorems for integrands satisfying the fast growth condition (Russian) Sibirsk. Mat. Zh. 46 (2005), 679-697; translation in Sib. Math. J. 46 (2005), 540-554.

[42] M. A. SYCHEV, Lower semicontinuity and relaxation for integral functionals with $p(x)$ and $p(x, u)$-growth (Russian) Sibirsk. Mat. Zh. 52 (2011), 1394-1413; translation in Sib. Math. J. 52 (2011), 1108-1123.

[43] M. A. SYCHEV, The theorem on convergence wuth a functional for integral functionals with $p(x)$ and $p(x, u)$-growth, Sib. Math. J. 53 (2012), 748-756.

[44] M. A. SYCHEV, Sets of lower semicontinuity and stability of integral functionals, J. Math. Pures Appl. (9) 84 (2005), 985-1014.

[45] M. A. SYCHEV, Weak convergence theory for strong materials with $p(x)$-growth, Dokl. Math. 87 (2013), 1-4.

[46] M. A. SYCHEV, Conditions on an integral functional that ensure the validity of the theory of weak convergence for it (Russian) Dokl. Akad. Nauk 439 (2011), 456-458; translation in Dokl. Math. 84 (2011), 522-524.

[47] M. A. SYCHEV, Characterization of homogeneous scalar variational problems solvable for all boundary data, Proc. Roy. Soc. Edinburgh Sect. A 130 (2000), 611-631.

[48] M. A. SycheV, A few remarks on differential inclusions, Proc. Roy. Soc. Edinburgh Sect. A 136 (2006), 649-668.

[49] S. S AKS, "Theory of the Integral", Hafner, New York, 1973.

[50] L. TONELl, Sur une methode directe du calcul des variations, Rend. Circ. Mat. Palermo 39 (1915), 233-264.

[51] L. TARTAR, Compensated compactness and applications to partial differential equations, In: "Nonlinear Analysis and Mechanics: Heriot-Watt Symposium", Vol. IV, Res. Notes in Math., Vol. 39, Pitman, Boston, Mass.-London, 1979, 136-212.

[52] V. Zhiкov, On some variational problems, Russian J. Math. Phys. 5 (1997), 105-116.

Sobolev Institute for Mathematics

Akad. Koptyug Prosp., 4

Novosibirsk 630090, Russia

masychev@math.nsc.ru

Novosibirsk State University

Novosibirsk 630090, Russia 